\title{
GAUSSIAN ESTIMATION OF PARAMETRIC SPECTRAL DENSITY WITH UNKNOWN POLE ${ }^{1}$
}

\author{
By L. GiRaitis, J. HidAlgo AND P. M. RoBinson ${ }^{2}$ \\ London School of Economics
}

\begin{abstract}
We consider a parametric spectral density with power-law behavior about a fractional pole at the unknown frequency $\omega$. The case of known $\omega$, especially $\omega=0$, is standard in the long memory literature. When $\omega$ is unknown, asymptotic distribution theory for estimates of parameters, including the (long) memory parameter, is significantly harder. We study a form of Gaussian estimate. We establish $n$-consistency of the estimate of $\omega$, and discuss its (non-standard) limiting distributional behavior. For the remaining parameter estimates, we establish $\sqrt{n}$-consistency and asymptotic normality.
\end{abstract}

1. Introduction. Cyclic behavior in covariance stationary time series is typically manifested in a pronounced peakedness in spectral density estimates. If the spectral density of a series $x_{t}, t=0, \pm 1, \pm 2, \ldots$, is estimated over the Nyqvist band $(-\pi, \pi]$, a peak at frequency $\omega \in(0, \pi]$ corresponds to a cycle of $2 \pi / \omega$. Various statistical models for such a phenomenon have been proposed. Two long-standing ones are of autoregressive (AR) and cosinusoidal type. For example, the $A R(2)$ model

$$
\left(1-\phi_{1} L-\phi_{2} L^{2}\right) x_{t}=\varepsilon_{t}, \quad t=0, \pm 1, \ldots,
$$

where $L$ is the lag operator and $\varepsilon_{t}$ is a sequence of uncorrelated and homoscedastic zero-mean random variables, has spectrum with peak at

$$
\omega=\arccos \left(\frac{\phi_{1}\left(\phi_{2}-1\right)}{4 \phi_{2}}\right)
$$

when the zeroes of $1-\phi_{1} z-\phi_{2} z^{2}$ are complex and $\left|\phi_{1}\left(\phi_{2}-1\right) / 4 \phi_{2}\right|<1$. On the other hand, a very simple cosinusoidal model is

$$
x_{t}=\alpha \cos (\omega t)+\beta \sin (\omega t),
$$

where $\alpha$ and $\beta$ are uncorrelated random variables with zero means and the same variance. Whereas (1.1) implies that $x_{t}$ has a spectral density that is analytic even at a peak, $x_{t}$ given by (1.2) has a spectral distribution function that jumps at $\omega$. The model (1.2) cannot describe real data, unlike the nonstationary-in-the-mean modification

$$
x_{t}=\alpha \cos (\omega t)+\beta \sin (\omega t)+u_{t},
$$

Received September 1999; revised May 2001

${ }^{1}$ Supported by ESRC Grant R000235892.

${ }^{2}$ Also supported by a Leverhulme Trust Personal Research Professorship.

AMS 2000 subject classifications. Primary 62M10; secondary 60G18.

Key words and phrases. Long range dependence, unknown pole. 
where $\alpha$ and $\beta$ are interpreted as fixed unknown constants and $u_{t}$ is an unobservable covariance stationary process with smooth spectral density. However, (1.1) and (1.2) constitute mathematically radically different descriptions of cyclic behavior within the stationary class, and help to motivate and place in perspective the model studied in the current paper.

An intermediate possibility between (1.1) and (1.2) is that $x_{t}$ has a spectral density with a pole at $\omega$. Denote by $f(\lambda)$ the spectral density of $x_{t}$, satisfying

$$
\gamma_{j} \stackrel{\text { def }}{=} E\left(x_{0} x_{j}\right)=\int_{-\pi}^{\pi} f(\lambda) \cos (j \lambda) d \lambda, \quad j=0, \pm 1, \ldots
$$

We are implicitly assuming here, as in (1.1) and (1.2), that $x_{t}$ has zero mean, but we will later indicate how our results apply in case $x_{t}$ has unknown mean. We say that $f(\lambda)$ has a (fractional) pole of order $\alpha \in(0,1)$ at $\omega$ if

$$
f(\lambda) \sim C|\lambda-\omega|^{-\alpha} \quad \text { as } \lambda \rightarrow \omega,
$$

where $C \in(0, \infty)$ and ' $\sim$ ' indicates that the ratio of left and right hand sides tends to 1 . In case $\omega=0,(1.4)$ is a familiar description of long-memory time series, so that for $\omega \in(0, \pi)(1.4)$ can be said to denote long-memory at a non-zero frequency. Parametric models for $f(\lambda)$, specifying $f$ for all $\lambda$ as a given function of unknown parameters and $\lambda$, and satisfying (1.4) for some $\omega \in(0, \pi)$, have been proposed by Hosking (1981), Andel (1986), Gray, Zhang and Woodward (1989) and others. The simplest of these modifies (1.1) to

$$
a(L ; \omega, d) x_{t}=\varepsilon_{t},
$$

where

$$
a(z ; \omega, d)=\left(1-2 z \cos \omega+z^{2}\right)^{d},
$$

and was termed a Gegenbauer model by Gray, Zhang and Woodward (1989). Writing $\sigma^{2}=\operatorname{Var}\left(\varepsilon_{t}\right)$, we deduce that, for $\lambda \in(-\pi, \pi]$,

$$
f(\lambda)=\frac{\sigma^{2}}{2 \pi}\left|a\left(e^{i \lambda} ; \omega, d\right)\right|^{-2}=\frac{\sigma^{2}}{2 \pi}\left|4 \sin \left(\frac{\lambda+\omega}{2}\right) \sin \left(\frac{\lambda-\omega}{2}\right)\right|^{-2 d} .
$$

When $d>0, f(\lambda)$ has a pole at $\lambda=\omega$ (and, when $\omega \neq 0$, at $-\omega$, as anticipated from symmetry). Moreover, when $\omega \in(0, \pi),(1.7)$ satisfies (1.4) with $\alpha=2 d$ and $C=\left(\sigma^{2} / 2 \pi\right)|2 \sin \omega|^{-2 d}$. When $\omega=0$ or $\pi$, (1.7) satisfies (1.4) with $\alpha=4 d$ and $C=\sigma^{2} / 2 \pi$, noting in case $\omega=\pi$ that $\left|\sin \frac{1}{2}(\lambda+\omega)\right|=\left|\sin \frac{1}{2}(\lambda-\omega)\right|$. Correspondingly, $x_{t}$ is covariance stationary for $d<1 / 2$ when $\omega \in(0, \pi)$ and for $d<1 / 4$ when $\omega=0$ or $\pi$. When $\omega=0, x_{t}$ is a standard long-memory model, FARIMA $(0,2 d, 0)$, though in this case the usual notation replaces $2 d$ by $d$. Note that a zero in $f(\lambda)$ occurs in (1.4) when $\alpha<0$ or in (1.7) when $d<0$, but we do not pursue this case. Hosking (1981), Gray, Zhang and Woodward (1989) extended (1.5) by replacing $\varepsilon_{t}$ by a covariance stationary and invertible autoregressive moving average (ARMA) process, when (1.4) is satisfied in the same way. Robinson (1994), Giraitis and Leipus (1995) considered more general models, providing spectral poles at several frequencies. They are motivated in part by seasonal processes, for which pole location is 
known. Hosoya (1997) established $\sqrt{n}$-consistency and asymptotic normality of Gaussian estimates of the remaining parameters in such models, extending work for the case of a known pole at zero frequency by Fox and Taqqu (1986), Dahlhaus (1989) and Giraitis and Surgailis (1990). In applications of non-seasonal, single-pole (in $(0, \pi])$ models such as $(1.5)$, however, it is likely that $\omega$ is unknown, as when investigating the length of a cycle in geophysical or macroeconomic time series.

The present paper proposes estimates based on a Gaussian objective function in case of parametric models satisfying (1.4) with unknown $\omega$ and studies their asymptotic statistical properties. The theory seems significantly harder than for the case of known $\omega$. The following section sets down the basic model with examples, and describes the parameter estimates, which are prompted by an approximate Gaussian likelihood, though we nowhere assume Gaussianity. In Section 3 the regularity conditions and limiting behavior of the parameter estimates are presented, with discussion, along with the main steps of the proof and a small numerical example, the remaining details appearing in Sections 4-7. Our main findings are that the estimates of $\omega$ are $n$-consistent [like those proposed by Hannan (1973a) for (1.3)], while the estimates of the remaining parameters are $\sqrt{n}$-consistent and have the same normal distribution as when $\omega$ is known.

2. Model and parameter estimates. We parameterize $f(\lambda)$ in the first place by writing

$$
f(\lambda)=\frac{\sigma_{0}^{2}}{2 \pi} k\left(\lambda ; \theta_{0}, \omega_{0}\right), \quad-\pi<\lambda \leq \pi,
$$

where $\sigma_{0}^{2}$ is an unknown positive scalar, $\theta_{0}$ is an unknown $p$-dimensional column vector, assumed to be in a compact set $\Theta \subset \mathbf{R}^{p}$, and $k(\lambda ; \theta, \omega)$ is a known function of $\lambda, \theta, \omega$, such that, for $\theta \in \Theta, \omega \in \Pi=[0, \pi]$,

$$
\begin{aligned}
k(\lambda ; \theta, \omega) & >0, \quad-\pi<\lambda \leq \pi, \\
\int_{-\pi}^{\pi} \log k(\lambda ; \theta, \omega) d \lambda & =0 .
\end{aligned}
$$

The zero subscripted quantities in (2.1) denote true values, $\theta$ and $\omega$ denoting any admissible values [so that $\alpha_{0}, \omega_{0}$, should replace $\alpha, \omega$ in (1.4), e.g.]. Following the discussion of Section 1 , we subdivide $\theta$ as $\theta=\left(\tau^{\prime}, \alpha\right)^{\prime}$ and correspondingly write $\Theta=\Theta_{\tau} \times \Theta_{\alpha}$ for compact sets $\Theta_{\tau}, \Theta_{\alpha}, \Theta_{\alpha} \subset[0,1]$. The $(p-1)$-vector $\tau$ is empty when $p=1$, in which case a particular $k(\lambda ; \theta, \omega)$ can be deduced from (1.7). For $p>1, \tau$ essentially describes short-range dependence, for example it can contain the coefficients of a stationary and invertible ARMA, so that we have the more general Gegenbauer model of Gray, Zhang and Woodward (1989), with

$$
k(\lambda ; \theta, \omega)=\left|\frac{a\left(e^{i \lambda} ; \omega, \alpha / 2\right) b\left(e^{i \lambda} ; \tau\right)}{c\left(e^{i \lambda} ; \tau\right)}\right|^{-2}, \quad-\pi<\lambda \leq \pi,
$$


where $a$ is given by (1.6), and with $\tau_{j}$ the $j$ th element of $\tau$,

$$
\begin{aligned}
& b(z ; \tau)=1-\sum_{j=1}^{p_{1}} \tau_{j} z^{j} 1_{\left\{1 \leq p_{1} \leq p-1\right\}}, \\
& c(z ; \tau)=1-\sum_{j=p_{1}+1}^{p-1} \tau_{j} z^{j-p_{1}} 1_{\left\{0 \leq p_{1} \leq p-2\right\}},
\end{aligned}
$$

where $1_{\{.\}}$is the indicator function, $0 \leq p_{1} \leq p-1$, all zeroes of $b(z ; \tau)$ and of $c(z ; \tau)$ are outside the unit circle, and $b(z ; \tau)$ and $c(z ; \tau)$ have no zeroes in common. More generally, we can consider models

$$
k(\lambda ; \theta, \omega)=\left|\frac{h(\lambda ; \tau)}{a\left(e^{i \lambda} ; \omega, \alpha / 2\right)}\right|^{2}, \quad-\pi<\lambda \leq \pi,
$$

where $h(\lambda ; \tau)$ is bounded and bounded away from zero. Condition (2.2) indicates that for a process with spectral density $\left(\sigma^{2} / 2 \pi\right) k(\lambda ; \theta, \omega)$, the free parameter $\sigma^{2}$ (functionally independent of $\theta$ and $\omega$ ) is the variance of the one-step-ahead best linear predictor [see Hannan (1970), pages 157-163]. In view of (2.2), which is satisfied by (2.3) and (2.5), we might consider, following Hannan $(1973 \mathrm{~b})$, estimating $\left(\theta_{0}^{\prime}, \omega_{0}\right)^{\prime}$ by

$$
\left(\begin{array}{c}
\tilde{\theta} \\
\tilde{\omega}
\end{array}\right)=\underset{\Theta \times \Pi}{\operatorname{argmin}} S(\theta, \omega)
$$

where

$$
S(\theta, \omega)=\tilde{n}^{-1} \sum_{j=0}^{\tilde{n}} \frac{I\left(\lambda_{j}\right)}{k\left(\lambda_{j} ; \theta, \omega\right)},
$$

$\tilde{n}=[n / 2]$ and we introduce the periodogram

$$
I\left(\lambda_{j}\right)=(2 \pi n)^{-1}\left|\sum_{t=1}^{n} x_{t} e^{i t \lambda_{j}}\right|^{2},
$$

evaluated at the Fourier frequencies $\lambda_{j}=2 \pi j / n$.

Under the conditions of Hannan (1973b), the objective function $S(\theta, \omega)$ approximates a Gaussian log-likelihood in the sense that $\left(\tilde{\theta}^{\prime}, \tilde{\omega}\right)^{\prime}$ has the same limit distribution as a Gaussian maximum likelihood estimate (though Hannan did not assume Gaussianity). However, Hannan's (1973b) conditions are not all satisfied due to the pole $\omega$ in $k(\lambda ; \theta, \omega)$. In case the true pole $\omega_{0}$ is known (e.g., it is taken for granted that $\omega_{0}=0$, as in standard long-memory models) then it is already known that the conditions of Hannan (1973b) can be suitably relaxed, as shown by Fox and Taqqu (1986), Dahlhaus (1989), Giraitis and Surgailis (1990) when $\omega_{0}=0$, and by Hosoya (1997) when $\omega_{0} \in(0, \pi]$, [though strictly these authors consider different approximations to the loglikelihood function from (2.6)]. Indeed the consistency proof of Hannan (without rates of convergence) still holds in case of known or unknown pole, the latter case having been considered by Giraitis and Leipus (1995). For limit distribution theory with a known pole $\omega_{0}$, the smoothness conditions of Hannan 
can be relaxed due to the "compensation" to $I(\lambda)$ afforded by the reciprocal of $k\left(\lambda ; \theta_{0}, \omega_{0}\right)$ near $\lambda=\omega_{0}$. When $\omega_{0}$ is unknown, derivation of limit distribution theory, and even rates of convergence, is significantly more difficult. An attempt at this was made, in the context of an alternative type of approximation to the Gaussian likelihood, by Chung (1996a, b); we shall briefly comment on Chung's treatment subsequently. Although $k(\lambda ; \theta, \omega)^{-1}=0$ at $\lambda=\omega$, the derivative $(\partial / \partial \omega) k^{-1}(\lambda ; \theta, \omega)$ is not well behaved near $\lambda=\omega$ in case of (2.3) and (2.5). For this reason we have chosen to study instead the estimate

$$
\left(\begin{array}{c}
\hat{\theta} \\
\hat{\omega}
\end{array}\right)=\underset{\Theta \times Q}{\operatorname{argmin}} S\left(\theta, \lambda_{q}\right)
$$

where $Q=Q_{n}=\{q: q=0,1, \ldots, \tilde{n}\}$. Thus minimization with respect to $\omega$ is now carried out over a discrete set. We could in fact consider a finer set, with spacing $\delta / n$ for arbitrary $\delta>0$, but it is essential that the intervals not be $o\left(n^{-1}\right)$ as $n \rightarrow \infty$. We can estimate $\sigma_{0}^{2}$ by

$$
\hat{\sigma}^{2}=S(\hat{\theta}, \hat{\omega}) .
$$

3. Regularity conditions and asymptotic properties. We introduce first the following assumptions, denoting by $\nabla_{\beta}$ the vector of partial derivatives $\partial / \partial \beta$, for a column vector or scalar $\beta$.

Assumption A.1. $\quad x_{t}$ has spectral density $f(\lambda)$ given by $(2.1),(2.5)$, that is

$$
f(\lambda)=\frac{\sigma_{0}^{2}}{2 \pi}\left|4 \sin \left(\frac{\lambda+\omega_{0}}{2}\right) \sin \left(\frac{\lambda-\omega_{0}}{2}\right)\right|^{-\alpha_{0}}\left|h\left(\lambda ; \tau_{0}\right)\right|^{2}, \quad-\pi<\lambda \leq \pi,
$$

where $\tau_{0}$ denotes the true value of $\tau$, and for $-\pi<\lambda \leq \pi, \tau \in \Theta_{\tau}, h(\lambda ; \tau)$ is even in $\lambda$ and bounded away from zero, and the derivatives $\nabla_{\tau} h(\lambda ; \tau), \nabla_{\lambda} h(\lambda ; \tau)$, $\nabla_{\tau} \nabla_{\lambda} h(\lambda ; \tau), \nabla_{\tau} \nabla_{\tau}^{\prime} h(\lambda ; \tau)$ are continuous.

Assumption A.2. (2.2) holds for all $\theta \in \Theta, \omega \in[0, \pi]$.

Assumption A.3.

$$
\inf _{\left(\theta^{\prime}, \omega\right) \in \Theta \times \Pi}(2 \pi)^{-1} \int_{-\pi}^{\pi} \frac{k\left(\lambda ; \theta_{0}, \omega_{0}\right)}{k(\lambda ; \theta, \omega)} d \lambda=1
$$

and the set

$$
\left\{\lambda: k(\lambda ; \theta, \omega) \neq k\left(\lambda ; \theta_{0}, \omega_{0}\right)\right\}, \quad\left(\theta^{\prime}, \omega\right) \neq\left(\theta_{0}^{\prime}, \omega_{0}\right)
$$

has positive Lebesgue measure. Also, the matrix

$$
\Omega=\left(w_{i j}\right)_{i, j=1, \ldots, p}=\frac{1}{4 \pi} \int_{-\pi}^{\pi} \nabla_{\theta} \log k\left(\lambda ; \theta_{0}, \omega_{0}\right) \nabla_{\theta}^{\prime} \log k\left(\lambda ; \theta_{0}, \omega_{0}\right) d \lambda
$$

is positive definite.

Assumption A.4. $\theta_{0}$ is an interior point of $\Theta$ and $\omega_{0} \in[0, \pi]$, such that $0<\alpha_{0}<1$ for $0<\omega_{0}<\pi$ and $0<\alpha_{0}<1 / 2$ for $\omega_{0}=0, \pi$. 
Assumption A.5. We have

$$
x_{t}=\sum_{j=0}^{\infty} \phi_{j} \varepsilon_{t-j}, \quad \sum_{j=0}^{\infty} \phi_{j}^{2}<\infty, \quad \phi_{0}=1,
$$

where $\left\{\varepsilon_{t}\right\}$ is ergodic and

$$
\begin{gathered}
E\left[\varepsilon_{t} \mid F_{t-1}\right]=0, \quad E\left[\varepsilon_{t}^{2} \mid F_{t-1}\right]=\sigma_{0}^{2} \quad \text { a.s., } \\
E\left[\varepsilon_{t}^{i} \mid F_{t-1}\right]=\mu_{i} \quad \text { a.s., } i=3,4,
\end{gathered}
$$

such that $\mu_{3}$ and $\mu_{4}$ are non-stochastic, and $F_{t}$ is the $\sigma$-algebra generated by $\varepsilon_{s}, s \leq t$; also, for some $\eta>0$,

$$
\max _{t} E\left|\varepsilon_{t}\right|^{4+\eta}<\infty .
$$

Assumption A.6. Uniformly in $\lambda \in[0, \pi] \backslash\left\{\omega_{0}\right\}$ the function $\phi(\lambda):=$ $\sum_{j=0}^{\infty} \phi_{j} e^{i j \lambda}$ has the property

$$
|(d / d \lambda) \phi(\lambda)|=O\left(|\phi(\lambda)|\left|\lambda-\omega_{0}\right|^{-1}\right) .
$$

Assumption A.1 covers a wide range of short memory spectral densities $h$, including both invertible ARMA ones and Bloomfield (1973) ones, though it also permits the modelling of processes with autocovariances that decay much more slowly than exponentially. We might call (3.1) a "generalized" Gegenbauer model. We have already discussed Assumption A.2, but add that in view of (1.6) and (2.5), it is equivalent to

$$
\int_{-\pi}^{\pi} \log h(\lambda ; \tau) d \lambda=0, \quad \tau \in \Theta_{\tau} .
$$

Assumption A.3 is an identifiability condition; note that in view of the structure of $a$, it will be implied if the set $\left\{\lambda: h(\lambda ; \tau) \neq h\left(\lambda ; \tau_{0}\right)\right\}, \tau \neq \tau_{0}$ has positive Lebesgue measure and the matrix

$$
\frac{1}{4 \pi} \int_{-\pi}^{\pi} \nabla_{\tau} \log h\left(\lambda ; \tau_{0}\right) \nabla_{\tau}^{\prime} \log h\left(\lambda ; \tau_{0}\right) d \lambda
$$

is positive definite. These conditions are satisfied in the ARMA case $h(\lambda ; \tau)=$ $\left|c\left(e^{i \lambda} ; \tau\right) / b\left(e^{i \lambda} ; \tau\right)\right|^{2}$, where $b(z ; \tau)$ and $c(z ; \tau)$ [see $(2.4)$ ] have no zeroes in common. Note that Assumption A.4 entails $\alpha_{0}>0$, which is essential for Assumption A.3 to hold, because $\omega_{0}$ is not identifiable when $\alpha_{0}=0$. In Assumption A.5, the normalization $\phi_{0}=1$ is consistent with Assumption A.2. Assumption A.5 is similar to ones used by Hannan (1973b) in Whittle estimation for short memory series, and on the other hand by Robinson (1995b) in narrow-band semiparametric Whittle estimation of long memory. However, Hannan's assumptions only extend to second moments in order to establish the Central Limit Theorem for estimators of $\tau_{0}$ in case $\alpha_{0}=0$, so that $f(\lambda)=\left(\sigma_{0}^{2} / 2 \pi\right) h\left(\lambda ; \tau_{0}\right)$, whereas Robinson allowed $\eta=0$ in (3.6). Assumption A.6 is like one used by Robinson (1995b). 
We also establish our results over a slightly different class than provided by Assumptions A.1, A.4:

Assumption A.1' $\quad x_{t}$ has spectral density given by (2.1) where

$$
k(\lambda ; \theta, \omega)= \begin{cases}|\lambda-\omega|^{-\alpha} g(\lambda ; \theta, \omega), & 0 \leq \lambda \leq \pi, \\ |\lambda+\omega|^{-\alpha} g(\lambda ; \theta, \omega), & -\pi<\lambda<0,\end{cases}
$$

such that for $-\pi<\lambda \leq \pi, \theta \in \Theta, \omega \in \Pi, g(\lambda ; \theta, \omega)$ is even in $\lambda$ and bounded and bounded away from zero, the derivatives $\nabla_{\theta} g(\lambda ; \theta, \omega), \nabla_{\theta} \nabla_{\theta}^{\prime} g(\lambda ; \theta, \omega)$ are continuous and bounded, $g(\lambda ; \theta, \omega)$ and $\nabla_{\theta} g(\lambda ; \theta, \omega)$ satisfy uniform Lipschitz conditions in $\omega$ of order greater than $1 / 2$, while, for $0<|\lambda|<\pi, \theta \in \Theta, \omega \in \Pi$, $\nabla_{\lambda} g(\lambda ; \theta, \omega)$ and $\nabla_{\lambda} \nabla_{\theta} g(\lambda ; \theta, \omega)$ are bounded.

We correspondingly replace Assumption A.4 by:

Assumption A.4 $4^{\prime} \quad \theta_{0}$ is an interior point of $\Theta$ such that $0<\alpha_{0}<1$, and $\omega_{0} \in[0, \pi]$.

Note that for $\omega \neq 0, \pi$, we can write the generalized Gegenbauer spectrum (2.5) in the form (3.7) by taking

$$
g(\lambda ; \theta, \omega)=\left\{\frac{|\lambda-\omega|^{\alpha}}{\left|a\left(e^{i \lambda} ; \omega, \alpha / 2\right)\right|^{2}}\right\}|h(\lambda ; \tau)|^{2}, \quad \lambda \in \Pi,
$$

with the corresponding expression for $-\pi<\lambda<0$, where the factor in braces tends to $(2 \sin \omega)^{-\alpha}$ as $\lambda \rightarrow \omega$, and is continuous but not differentiable at $\lambda=$ $0, \pi$ because of the inevitable evenness and periodicity of spectral densities, explaining our avoidance of differentiability in $\lambda$ at these frequencies. In other words, if $g(\lambda ; \theta, \omega)$ were differentiable at $\lambda=0, \pi$, then $k(\lambda ; \theta, \omega)$ would not be, and the Gegenbauer model (2.3), for example, would be excluded. On the other hand, Assumption A.1' also includes models for which $g(\lambda ; \theta, \omega)$ is everywhere smooth, such as when

$$
k(\lambda ; \theta, \omega)=c(\theta, \omega)|\lambda-\omega|^{-\alpha}, \quad \lambda \in \Pi,
$$

with $c(\theta, \omega)=\exp \{(\alpha / \pi)[\omega(\log \omega-1)+(\pi-\omega)(\log (\pi-\omega)-1)]\}$, so that Assumption A.2 is satisfied. Moreover, under Assumption A.1' we constrain $\alpha$ to $(0,1)$, not $(0,1 / 2)$ at $\omega=0, \pi$, so we avoid this type of discontinuity. Notice that from (1.7), $\left|a\left(e^{i \lambda} ; \omega, \alpha / 2\right)\right|^{-2} \sim(2 \sin \omega)^{-\alpha}|\lambda-\omega|^{-\alpha}$ as $\lambda \rightarrow \omega$ for $\omega \neq 0, \pi$, so that $\left|a\left(e^{i \lambda} ; \omega, \alpha / 2\right)\right|^{-2}$ and $|\lambda-\omega|^{-\alpha}$ behave similarly around the pole. The model (3.8) was mentioned by Hosoya (1997). One notable feature of (3.8) is that besides the pole at $\lambda=\omega$, intended by the modeller, it entails lack of differentiability in $\lambda$ at $\lambda=0, \pi$, which, alongside the infinite differentiability of $k$ at all $\lambda \neq 0, \omega, \pi$, is probably not a feature that a modeller would intend. On the other hand, models for short memory series with non-differentiable peaks or troughs at frequencies $0, \pi$, were considered by Robinson (1978). 
To discuss further the distinction between A.1, A.4 and A.1', A.4', note that we can also write (2.5) as

$$
k(\lambda ; \theta, \omega)= \begin{cases}|\lambda-\omega|^{-\alpha}|\lambda+\omega|^{-\alpha} g_{1}(\lambda ; \theta, \omega), & 0 \leq \lambda \leq \pi / 2, \\ |\lambda-\omega|^{-\alpha}|\lambda+\omega-2 \pi|^{-\alpha} g_{2}(\lambda ; \theta, \omega), & \pi / 2<\lambda \leq \pi,\end{cases}
$$

where

$$
\begin{aligned}
& g_{1}(\lambda ; \theta, \omega)=\left|\frac{(\lambda-\omega)(\lambda+\omega)}{4 \sin \left(\frac{\lambda+\omega}{2}\right) \sin \left(\frac{\lambda-\omega}{2}\right)}\right|^{\alpha}|h(\lambda ; \tau)|^{2}, \\
& g_{2}(\lambda ; \theta, \omega)=\left|\frac{(\lambda-\omega)(\lambda+\omega-2 \pi)}{4 \sin \left(\frac{\lambda+\omega}{2}\right) \sin \left(\frac{\lambda-\omega}{2}\right)}\right|^{\alpha}|h(\lambda ; \tau)|^{2} .
\end{aligned}
$$

From (3.9) it follows that (3.7) holds with

$$
g(\lambda ; \theta, \omega)= \begin{cases}|\lambda+\omega|^{-\alpha} g_{1}(\lambda ; \theta, \omega), & 0 \leq \lambda \leq \pi / 2, \\ |\lambda+\omega-2 \pi|^{-\alpha} g_{2}(\lambda ; \theta, \omega), & \pi / 2<\lambda \leq \pi .\end{cases}
$$

Although the functions $g_{1}, g_{2}$ satisfy Assumptions A.1' on $g$, (3.10) does not satisfy these assumptions, since it is unbounded at $\lambda=\omega$ for $\omega=0, \pi$. We shall use in the proofs the fact that the Gegenbauer density $k$ can be written in the form (3.9); the fact that in this expression $g_{1}, g_{2}$ satisfy Assumption A. $1^{\prime}$ on $g$; and the fact that if $\omega \in[\delta, \pi-\delta]$ where $\delta>0$, i.e. when $\omega$ is separated from $0, \pi$, the function $g$, given by (3.10) satisfies Assumption A. $1^{\prime}$ on $g$.

For brevity we say that Assumptions A are satisfied if either A.1-A.6 hold or A.1', A.2, A.3, A.4', A.5 and A.6 hold.

TheOREm 3.1. Under Assumptions A, as $n \rightarrow \infty$,

$$
\widehat{\theta}-\theta_{0}=O_{P}\left(n^{-1 / 2}\right), \quad \widehat{\omega}-\omega_{0}=O_{P}\left(n^{-1}\right) .
$$

Proof. It suffices to show that for all $\varepsilon>0$ we can choose $K=K_{\varepsilon}$ such that

$$
P\left\{n|| \widehat{\theta}-\theta_{0} \|^{2} \geq K\right\}+P\left\{n\left|\widehat{\omega}-\omega_{0}\right| \geq 2 \pi(K+1)\right\} \leq \varepsilon
$$

for $n$ sufficiently large, where $\|$.$\| denotes Euclidean norm. Define q_{0}=q_{0 n}=$ $\arg \min _{Q}\left|\omega_{0}-\lambda_{q}\right|$ so that $\left|q_{0}-\frac{n \omega_{0}}{2 \pi}\right| \leq \frac{1}{2}$. If there are two such $q$, define $q_{0}$ as the smaller. Thus the left side of (3.11) is bounded by twice

$$
P\left\{u_{n}(\hat{\psi}) \geq K\right\},
$$

where $\psi=\left(\theta^{\prime}, \omega\right)^{\prime}, \hat{\psi}=\left(\hat{\theta}^{\prime}, \hat{\omega}\right)^{\prime}$ and $u_{n}(\psi)=n\left\|\theta-\theta_{0}\right\|^{2}+|\bar{q}|$ with $\bar{q}=q-q_{0}$. By a standard type of argument for proving consistency of implicitly defined 
extremum estimates, (3.12) is bounded by

$$
\begin{aligned}
& P\left(\inf _{\psi \in \Psi_{K}}\left\{S(\psi)-S\left(\psi_{0}\right)\right\} \leq 0\right) \\
& \quad=P\left(\inf _{\psi \in \Psi_{K}}\left\{u_{n}(\psi)^{-1}\left(S(\psi)-S\left(\psi_{0}\right)\right)\right\} \leq 0\right),
\end{aligned}
$$

where $S(\psi)=S(\theta, \omega)$ and $\Psi_{K}=\left\{\psi: \theta \in \Theta, q \in Q, u_{n}(\psi) \geq K\right\}$. Now (3.13) is bounded by

$$
P\left(\sup _{\Psi_{K}}\left|u_{n}(\psi)^{-1} U_{n}(\psi)\right|+\sup _{\Psi_{K}}\left|u_{n}(\psi)^{-1} V_{n}(\psi)\right|+K^{-1} \inf _{\Psi_{K}} u_{n}(\psi)^{-1} T_{n}(\psi)\right),
$$

where

$$
\begin{gathered}
T_{n}(\psi)=\sum_{j\left(q, q_{0}\right)}\left\{\frac{k_{j}\left(\psi_{0}\right)}{k_{j}(\psi)}-1\right\}, \quad V_{n}(\psi)=1-\frac{2 \pi I_{q_{0}}}{\sigma_{0}^{2} k_{q_{0}}(\psi)}, \\
U_{n}(\psi)=\sum_{j\left(q_{0}\right)}{ }^{\prime}\left\{\frac{k_{j}\left(\psi_{0}\right)}{k_{j}(\psi)}-1\right\}\left\{\frac{2 \pi I_{j}}{\sigma_{0}^{2} k_{j}\left(\psi_{0}\right)}-1\right\}
\end{gathered}
$$

and $I_{j}=I\left(\lambda_{j}\right), k_{j}(\psi)=k\left(\lambda_{j} ; \psi\right), \sum_{j(q)}^{\prime}=\sum_{j=0, j \neq q}^{\tilde{n}}, \sum_{j\left(q, q_{0}\right)}^{\prime}=\sum_{j=0, j \neq q, q_{0}}^{\tilde{n}}$, so that

$$
S\left(\psi_{0}\right)-S(\psi)=\frac{\sigma_{0}^{2}}{2 \pi \tilde{n}}\left\{-U_{n}(\psi)+V_{n}(\psi)-T_{n}(\psi)-1_{\left\{q=q_{0}\right\}}\right\} .
$$

It is shown in Lemmas 6.1 and 6.2 below that as $n \rightarrow \infty, K \rightarrow \infty$,

$$
E \sup _{\psi \in \Psi_{K}}\left|u_{n}(\psi)^{-1} U_{n}(\psi)\right| \rightarrow 0, \quad E \sup _{\psi \in \Psi_{K}}\left|u_{n}(\psi)^{-1} V_{n}(\psi)\right| \rightarrow 0
$$

whereas by Lemma 7.1 below, for some $c>0$,

$$
\inf _{\psi \in \Psi_{K}}\left(u_{n}(\psi)^{-1} T_{n}(\psi)\right) \geq c
$$

where $c$ does not depend on $K, n$. Thus, by Markov's inequality (3.14) is bounded by

$$
\begin{aligned}
& \frac{\left.E\left(\sup _{\Psi_{K}}\left|u_{n}(\psi)^{-1} U_{n}(\psi)\right|+\sup _{\Psi_{K}} \mid u_{n}(\psi)^{-1} V_{n}(\psi)\right) \mid+K^{-1}\right)}{\inf _{\Psi_{K}}\left(u_{n}(\psi)^{-1} T_{n}(\psi)\right)} \rightarrow 0 \\
&(n \rightarrow \infty, K \rightarrow \infty) .
\end{aligned}
$$

TheOREM 3.2. Under Assumptions A, as $n \rightarrow \infty$,

$$
n^{1 / 2}\left(\widehat{\theta}-\theta_{0}\right) \stackrel{d}{\Rightarrow} N\left(0, \Omega^{-1}\right)
$$

PRoOF. From Theorem 3.1 and the fact that $\theta_{0}$ is an interior point of $\Theta$, for $n$ sufficiently large

$$
0=\nabla_{\theta} S(\widehat{\psi})=\nabla_{\theta} S\left(\theta_{0}, \hat{\omega}\right)+M\left(\widehat{\theta}-\theta_{0}\right),
$$


where the $i$ th row of the $p \times p$ matrix $M$ is the $i$ th row of $\nabla_{\theta} \nabla_{\theta}^{\prime} S(\theta, \widehat{\omega})$ evaluated at $\theta^{(i)}$, where $\left\|\theta^{(i)}-\theta_{0}\right\| \leq\left\|\widehat{\theta}-\theta_{0}\right\|$. Since $\left\{\varepsilon_{t}\right\}$ is ergodic, by a well-known argument [see, e.g., Theorem 3.5.8 of Stout (1974)], the moving average sequence $\left\{x_{t}\right\}$ (3.5) is also ergodic. Therefore from Lemma 1 of Hannan (1973b) and Assumptions A, it follows that

$$
M \stackrel{P}{\Rightarrow} \pi^{-1} \sigma_{0}^{2} \Omega .
$$

To complete the proof it remains to prove that

$$
n^{1 / 2} \nabla_{\theta} S\left(\theta_{0}, \widehat{\omega}\right) \stackrel{d}{\Rightarrow} N\left(0, \pi^{-2} \sigma_{0}^{4} \Omega\right),
$$

which is shown in Lemma 5.2 below.

Theorem 3.2 indicates that the estimates of the short and long memory parameters $\tau_{0}$ and $\alpha_{0}$ have the same limit distribution when $\omega_{0}$ is unknown as when it is known. In order to use the theorem in conducting inference on $\theta_{0}$ we might consistently estimate $\Omega$ by

$$
\hat{\Omega}=n^{-1} \sum_{j(\hat{q})}^{\prime} \nabla_{\theta} \log k\left(\lambda_{j} ; \hat{\theta}, \hat{\omega}\right) \nabla_{\theta}^{\prime} \log k\left(\lambda_{j} ; \hat{\theta}, \hat{\omega}\right) .
$$

Given Theorem 3.1, it is straightforward to show that $n^{1 / 2}\left(\widehat{\sigma}^{2}-\sigma_{0}^{2}\right) \stackrel{d}{\Rightarrow}$ $N\left(0,2 \sigma_{0}^{4}+\kappa_{4}\right)$ as $n \rightarrow \infty$ (where $\kappa_{4}$ is the 4th cumulant of $\varepsilon_{t}$ ), as in Whittle estimation of other models. by

We now allow that $E x_{t}=\mu$ is unknown, $\mu \in \mathbf{R}$, estimating $\left(\theta_{0}^{\prime}, \omega_{0}\right)^{\prime}$ instead

$$
\left(\begin{array}{c}
\hat{\theta}^{*} \\
\hat{\omega}^{*}
\end{array}\right)=\arg \min _{\Theta \times Q} S^{*}\left(\theta, \lambda_{q}\right) \quad \text { where } S^{*}(\theta, \omega)=\tilde{n}^{-1} \sum_{j=1}^{\tilde{n}} \frac{I\left(\lambda_{j}\right)}{k\left(\lambda_{j} ; \theta, \omega\right)} .
$$

THEOREM 3.3. Under Assumptions A, with $x_{t}=\mu+\sum_{j=0}^{\infty} \phi_{j} \varepsilon_{t-j}$ replacing the representation for $x_{t}$ in A.5, the results of Theorems 3.1 and 3.2 remain valid for the modified estimate $\left(\hat{\theta}^{* \prime}, \hat{\omega}^{*}\right)^{\prime}$.

Proof. As is well known, for the Fourier frequencies $\lambda_{j}, j=1, \ldots, \tilde{n}$, we can write

$$
I\left(\lambda_{j}\right)=(2 \pi n)^{-1}\left|\sum_{t=1}^{n}\left(x_{t}-E x_{t}\right) e^{i t \lambda_{j}}\right|^{2}
$$

since $I\left(\lambda_{j}\right)$ is invariant to location shift in $x_{t}$ for such $j$. Hence

$$
S^{*}(\theta, \omega)=S(\theta, \omega)-\tilde{n}^{-1} R_{n}(\theta, \omega),
$$

where $R_{n}(\theta, \omega)=k(0 ; \theta, \omega)^{-1} I(0)$. Similarly to the proof of Lemma 6.2 it can be shown that

$$
E \sup _{\psi \in \Psi_{K}}\left|u_{n}(\psi)^{-1} R_{n}(\theta, \omega)\right| \leq C K^{-\eta}
$$


for some $\eta>0$ where $C>0$ does not depend on $n, \eta$ and

$$
\begin{aligned}
E \sup _{\psi \notin \Psi_{K}}\left\|n^{-1} \nabla_{\theta} R_{n}(\theta, \omega)\right\| & =o\left(n^{-1 / 2}\right), \\
E \sup _{\psi \notin \Psi_{K}}\left|n^{-1} R_{n}(\theta, \omega)\right| & =o\left(n^{-1 / 2}\right) \quad(n \rightarrow \infty) .
\end{aligned}
$$

These relations imply that the term $\tilde{n}^{-1} R_{n}(\theta, \omega)$ in (3.23) is negligible. Namely, $S(\theta, \omega)$ approximates $S^{*}(\theta, \omega)$ and the same method of proof as in Theorems 3.1-3.2 can be applied.

Chung (1996a, b) claims to have established the limit distribution of related estimates of both $\theta_{0}$ and $\omega_{0}$ in the context of the Gegenbauer model (2.3). Chung uses a conditional sum of squares method suggested by Gray, Zhang and Woodward (1989) in the same setting, and employed earlier by Box and Jenkins (1971) for ARMA models. This involves a time domain approximation to the Gaussian log likelihood, in which the conditional mean and variance of $x_{t}$ given $x_{s}, 1 \leq s<t$ are replaced respectively by the conditional expectation given $x_{s},-\infty<s<t$ with $x_{s}$ then set to zero for $s \leq 0$, and the innovation variance. Our frequency domain approximation to the Gaussian log likelihood is proposed in part for computational reasons, because the functional form of the spectral density is typically of simple form and immediately identifiable, as in the Gegenbauer case, whereas time domain features such as AR coefficients are relatively cumbersome, while our approach can also make direct use of the fast Fourier transform. Though the limit distribution Chung states for his estimate of $\theta_{0}$ is identical to that of ours, we are unable to check various details of his proofs of limit theory for estimates of $\theta_{0}$ or $\omega_{0}$. Perhaps, most notably, Chung claims that consistency of his estimates follows from the property that the expectation of his log likelihood approximation has zero derivative at the true parameter point. This property is insufficient, however, especially in the context of implicitly defined extremum estimates, where an initial consistency proof is an essential first step to deriving limit distribution theory, and indeed it is the proof of consistency that is the most challenging problem in the present situation due to the different rates of convergence of the estimates of $\theta_{0}$ and $\omega_{0}$, as we believe the proof of our Theorem 3.1 illustrates.

In the semiparametric context (1.4) there are known partial answers. Yajima (1996), in Gaussian case, and Hidalgo (1999), in the linear process case, have proposed estimators for $\omega_{0}$ that are $n^{\delta}$-consistent for any $\delta<1$. Though Yajima (1996) did not obtain the asymptotic distribution, Hidalgo (1999) established asymptotic normality and suggested an estimator for $\theta_{0}$ having the same rate of convergence and limiting distribution as in case of known $\omega_{0}$.

Theorem 3.1 establishes $n$-consistency of $\hat{\omega}$. We are unable to derive its limit distribution. In fact we believe that none exists, due to the fact that $\hat{\omega}$ minimizes $S(\theta, \omega)$ not over the interval $[0, \pi]$ but over the grid $Q$, with mesh $2 \pi / n$. If $\omega_{0} \neq 0$, the Fourier frequency $\lambda_{q_{0}}$, closest to $\omega_{0}$, satisfies $\left|\lambda_{q_{0}}-\omega_{0}\right| \leq$ $2 \pi / n$ but the limit $n\left|\lambda_{q_{0}}-\omega_{0}\right| \quad(n \rightarrow \infty)$ does not exist. To explain the problem 
more precisely, note that

$$
S\left(\hat{\theta}, \lambda_{\hat{q}}\right)-S\left(\theta_{0}, \lambda_{q_{0}}\right)=\left(S\left(\theta_{0}, \lambda_{\hat{q}}\right)-S\left(\theta_{0}, \lambda_{q_{0}}\right)\right)+(1 / 2)\left(\hat{\theta}-\theta_{0}\right)^{\prime} M\left(\hat{\theta}-\theta_{0}\right) .
$$

By Theorem 3.2 and (3.21), the limit $\tilde{n}\left(\hat{\theta}-\theta_{0}\right)^{\prime} M\left(\hat{\theta}-\theta_{0}\right) \stackrel{d}{\Rightarrow}\left(\sigma_{0}^{2} / 2 \pi\right) Z^{\prime} \Omega Z$ as $n \rightarrow \infty$ exists and does not depend on $\lambda_{q}$, $\omega$, where $Z \sim N\left(0, \Omega^{-1}\right)$. Therefore, by (3.24), the limit distribution of $\hat{q}$ can be defined as $\operatorname{argmin}_{q} \lim _{n \rightarrow \infty} V_{n}(q)$ where $V_{n}(q)=\tilde{n}\left(S\left(\theta_{0}, \lambda_{q}\right)-S\left(\theta_{0}, \lambda_{q_{0}}\right)\right)$. Write

$$
V_{n}(q)=-\sum_{j\left(q_{0}\right)}{ }^{\prime} k_{j}^{-1}\left(\psi_{0}\right) I_{j}+\sum_{j(q)}^{\prime} k_{j}^{-1}\left(\theta_{0}, \lambda_{q}\right) I_{j}=\sum_{j\left(q_{0}\right)}{ }^{\prime} d_{n, j} z_{n}(j)+k_{q_{0}}^{-1}\left(\theta_{0}, \lambda_{q}\right) I_{q_{0}}
$$

where $d_{n, j}=\sigma_{0}^{2}\left(k_{j}^{-1}\left(\theta_{0}, \lambda_{q}\right) k_{j}\left(\theta_{0}, \lambda_{q_{0}}\right)-1\right), \quad z_{n}(j)=\left(\sigma_{0}^{2} k_{j}\left(\theta_{0}, \lambda_{q_{0}}\right)\right)^{-1} I_{j}$. Note that for fixed finite $j$, as $n \rightarrow \infty, d_{n, j} \sim \tilde{d}_{n, j}=\sigma_{0}^{2}\left(\left|(j-q) /\left(j-q_{0}\right)\right|^{\alpha_{0}}-1\right)$. If the limit $z_{n}(j) \Rightarrow z(j)$ exists we would expect that $V_{n}(q) \sim V(q)=$ $\sum_{j=0: j \neq q_{0}}^{\infty} d_{j} z(j)+k_{q_{0}}^{-1}\left(\theta_{0}, \lambda_{q}\right) I_{q_{0}}$. However, in case $\omega_{0} \neq 0$, even the limit of $E z_{n}(j)$ does not exist. Indeed,

$$
E z_{n}(j)=\frac{k_{j}\left(\theta_{0}, \omega_{0}\right)}{k_{j}\left(\psi_{0}\right)}\left(\sigma_{0}^{2} k_{j}\left(\theta_{0}, \omega_{0}\right)\right)^{-1} E I_{j} .
$$

By Robinson (1995a), $\left(\sigma_{0}^{2} k_{j}\left(\theta_{0}, \omega_{0}\right)\right)^{-1} E I_{j}$ has finite limit as $n \rightarrow \infty$ but

$$
k_{j}\left(\theta_{0}, \omega_{0}\right) / k_{j}\left(\psi_{0}\right) \sim\left|\left(\lambda_{j}-\omega_{0}\right) /\left(\lambda_{j}-\lambda_{q_{0}}\right)\right|^{\alpha_{0}}=\left|1+\left(2 \pi n\left(\lambda_{q_{0}}-\omega_{0}\right)\right) /\left(j-q_{0}\right)\right|^{\alpha_{0}}
$$

does not converge since as $n \rightarrow \infty$ the limit of $n\left(\lambda_{q_{0}}-\omega_{0}\right)$ does not exist.

To illustrate the finite-sample performance of our procedure, a small Monte Carlo experiment was carrried out by Dr. Gilles Teyssiére on the basis of the simple Gegenbauer model (1.5), with $\varepsilon_{t}$ Gaussian, $d=0.4$ and $\omega=$ $0.25,0.5,1.0,2.0,2.5,3.0$. 5,000 replications of sample sizes $n=64$ and 256 were generated using 3,000 presample innovations and truncating the MA expansion at 3, 000 terms. Monte Carlo bias and standard deviation (SD) of $\widehat{\omega}$ and $\widehat{d}=\widehat{\alpha} / 2$ are reported in Tables 1 and 2. The bias in $\widehat{\omega}$ seems fairly uniform across $\omega$, whereas the bias of $\widehat{d}$ is noticeably greater near $\omega=\pi / 2$. Biases significantly decline as $n$ increases, while in a more specific way the asymptotic theory, which predicts that $\operatorname{SD}(\widehat{d})$ and $\operatorname{SD}(\widehat{\omega})$ should be respectively doubled and quadrupled going from $n=256$ to $n=64$, is fairly well reflected in Table 1.

4. Central limit theorem for weighted sums of periodograms. Throughout this section we assume that $\left\{x_{t}\right\}$ satisfies Assumptions A, so it has spectral density

$$
f(\lambda)=\frac{\sigma_{0}^{2}}{2 \pi}\left|\lambda-\omega_{0}\right|^{-\alpha} g(\lambda), \quad 0 \leq \lambda \leq \pi
$$

where $\sigma_{0}^{2}=E \varepsilon_{0}^{2}$ and $g(\lambda)$ is bounded away from infinity and zero and has bounded derivative $(\partial / \partial \lambda) g(\lambda)$ uniformly over $0<\lambda<\pi$. However, whereas 
TABLE 1

\begin{tabular}{lccccccccc}
\hline \multicolumn{3}{c}{$n=\mathbf{6 4}$} & \multicolumn{7}{c}{$\boldsymbol{n}=\mathbf{2 5 6}$} \\
\hline $\boldsymbol{\omega}$ & $\mathbf{B I A S}(\hat{\boldsymbol{\omega}})$ & $\mathbf{S D}(\hat{\boldsymbol{\omega}})$ & $\mathbf{B I A S}(\hat{\boldsymbol{d}})$ & $\mathbf{S D}(\hat{\boldsymbol{d}})$ & $\boldsymbol{\omega}$ & $\mathbf{B I A S}(\hat{\boldsymbol{\omega}})$ & $\mathbf{S D}(\hat{\boldsymbol{\omega}})$ & $\operatorname{BIAS}(\hat{\boldsymbol{d}})$ & $\mathbf{S D}(\hat{\boldsymbol{d}})$ \\
\hline 0.25 & -.001 & .085 & -.013 & .074 & 0.25 & -.002 & .019 & -.006 & .031 \\
0.5 & -.014 & .081 & -.042 & .080 & 0.5 & -.003 & .020 & -.002 & .037 \\
1.0 & -.023 & .110 & -.075 & .111 & 1.0 & .001 & .020 & -.011 & .049 \\
1.5 & -.015 & .119 & -.108 & .138 & 1.5 & -.002 & .019 & -.031 & .059 \\
2.0 & .000 & .107 & -.085 & .127 & 2.0 & .000 & .022 & -.009 & .055 \\
2.5 & .000 & .089 & -.050 & .096 & 2.5 & .002 & .018 & -.016 & .039 \\
3.0 & -.026 & .078 & -.054 & .079 & 3.0 & -.004 & .019 & -.011 & .031 \\
\hline
\end{tabular}

Assumptions A entail $\alpha \in(0,1)$, we allow in the current section also for $\alpha=0$. Note that Assumption A.2 does not need to be imposed, however.

We now prove a central limit theorem for the sums

$$
S_{n}:=\sum_{j\left(q_{0}\right)}{ }^{\prime} b_{j}\left(\frac{I_{j}}{f\left(\lambda_{j}\right)}-1\right)
$$

with real weights $b_{j} \equiv b_{n, j}, j=0, \ldots, \tilde{n}$, the conditions on which are formulated in terms of

$$
n_{1}(b):=n^{-1 / 2} \sum_{j\left(q_{0}\right)}^{\prime} b_{j}, \quad n_{2}(b):=\sum_{j\left(q_{0}\right)}{ }^{\prime} b_{j}^{2}
$$

and

$$
n_{3}(b, \gamma):=\sum_{j=-1: j \neq q_{0}, q_{0}-1}^{\tilde{n}}\left|b_{j}-b_{j+1}\right|\left|j-q_{0}\right|^{\gamma} \quad(\gamma>0),
$$

setting $b_{-1}=b_{\tilde{n}+1}=0$.

LEMMA 4.1. Suppose that as $n \rightarrow \infty$

$$
n_{1}(b) \rightarrow v_{1}<\infty, \quad n_{2}(b) \rightarrow v_{2}<\infty \quad \text { and } \quad n_{3}(b, \gamma) \rightarrow 0
$$

for some $\gamma>1 / 3$. Then $S_{n}$, given by (4.2), satisfies

$$
S_{n} \stackrel{d}{\Rightarrow} N\left(0, \sigma^{2}\right)
$$

where $\sigma^{2}=v_{2}+v_{1}^{2} \sigma_{0}^{-4} \kappa_{4}$.

The proof of Lemma 4.1 is technical and is reserved for the next section.

We consider now a special case of the weights $b_{j}$.

THEOREM 4.2. Let

$$
b_{j}=n^{-1 / 2} h_{n}\left(\lambda_{j}\right), \quad j=0, \ldots, \tilde{n}
$$

where $h_{n}(\lambda), \lambda \in[0, \pi], n \geq 1$ are real valued functions. Suppose there exist $C>0, K>0,0 \leq \alpha^{\prime}<1 / 2$ and $0 \leq \gamma^{\prime}<3 / 2$, independent of $n$, such that for 
all sufficiently large $n$ :

(c1.) $\left|h_{n}(\lambda)\right| \leq C\left|\lambda-\omega_{0}\right|^{-\alpha^{\prime}}$ uniformly in $\left|\lambda-\omega_{0}\right| \geq \pi / n$,

(c2.) $\left|(d / d \lambda) h_{n}(\lambda)\right| \leq C\left|\lambda-\omega_{0}\right|^{-\gamma^{\prime}}$ uniformly in $\left|\lambda-\omega_{0}\right| \geq K / n, 0<\lambda<\pi$, and also,

(c3.) $\lim _{n \rightarrow \infty} h_{n}(\lambda)=h(\lambda)$ exists for $\lambda \neq \omega_{0}$.

Then (4.4) holds with

$$
v_{1}=(2 \pi)^{-1} \int_{0}^{\pi} h(\lambda) d \lambda, \quad v_{2}=(2 \pi)^{-1} \int_{0}^{\pi} h^{2}(\lambda) d \lambda .
$$

Proof. By Lemma 4.1, it suffices to show that as $n \rightarrow \infty$ the weights $b_{j}=n^{-1 / 2} h_{n}\left(\lambda_{j}\right)$ satisfy (4.3). We prove first that $n_{3}(b, \gamma) \rightarrow 0$ for any $\gamma \in$ $(1 / 3,1 / 2)$. By $(\mathrm{c} 1)$, with $b_{-1}=b_{\tilde{n}+1}=0$,

$$
\begin{aligned}
n_{3}(b, \gamma) & =n^{-1 / 2} \sum_{j=0:\left|j-q_{0}\right| \geq 2 K}^{\tilde{n}-1}\left|h_{n}\left(\lambda_{j}\right)-h_{n}\left(\lambda_{j+1}\right)\right|\left|j-q_{0}\right|^{\gamma}+o(1) \\
& \leq C n^{-3 / 2} \sum_{j=0:\left|j-q_{0}\right| \geq 2 K}^{n}\left(n /\left|j-q_{0}\right|\right)^{\gamma^{\prime}}\left|j-q_{0}\right|^{\gamma}+o(1)=o(1)
\end{aligned}
$$

since by (c2),

$$
\left|h_{n}\left(\lambda_{j}\right)-h_{n}\left(\lambda_{j+1}\right)\right| \leq C n^{-1} \sup _{\xi \in\left[\lambda_{j}, \lambda_{j+1}\right]}\left|h_{n}^{\prime}(\xi)\right| \leq C n^{-1}\left|\lambda_{j}-\lambda_{q_{0}}\right|^{-\gamma^{\prime}} .
$$

Convergence of the sums $n_{1}(b)=n^{-1} \sum_{j\left(q_{0}\right)}^{\prime} h_{n}\left(\lambda_{j}\right) \rightarrow v_{1}, n_{2}(b)=n^{-1} \sum_{j\left(q_{0}\right)}^{\prime}$ $h_{n}^{2}\left(\lambda_{j}\right) \rightarrow v_{2}$ follows by Lemma 5.3 below, because (c1)-(c3) imply $h_{n}^{2}(\lambda) \leq$ $C\left|\lambda-\omega_{0}\right|^{-2 \alpha^{\prime}}$ uniformly in $\left|\lambda-\omega_{0}\right| \geq \pi / n$ and $\left|(d / d \lambda) h_{n}^{2}(\lambda)\right| \leq C\left|\lambda-\omega_{0}\right|^{-\alpha^{\prime}-\gamma^{\prime}}$ uniformly in $\left|\lambda-\omega_{0}\right| \geq K / n$.

We now provide an auxiliary lemma on the approximation of normalized periodograms. Denote $I_{j, \varepsilon}=(2 \pi n)^{-1}\left|\sum_{t=1}^{n} \varepsilon_{t} e^{i t \lambda_{j}}\right|^{2}$ and write

$$
\begin{aligned}
& \xi_{j, n}^{(1)}=f^{-1}\left(\lambda_{j}\right) I_{j}-1 ; \quad \xi_{j, n}^{(2)}=2 \pi I_{j, \varepsilon} / \sigma_{0}^{2}-1 ; \\
& \xi_{j, n}^{(3)}=f^{-1}\left(\lambda_{j}\right) I_{j}-2 \pi I_{j, \varepsilon} / \sigma_{0}^{2} .
\end{aligned}
$$

Define for $i=1,2,3$,

$$
Z_{j, n}^{(i)}= \begin{cases}\sum_{\ell=q_{0}+1}^{j} \xi_{\ell, n}^{(i)}, & j=q_{0}+1, \ldots, \tilde{n}, \\ \sum_{\ell=j+1}^{q_{0}-1} \xi_{\ell, n}^{(i)}, & j=-1, \ldots, q_{0}-2,\end{cases}
$$

recalling that $\lambda_{q_{0}}$ is the closest Fourier frequency to $\omega_{0}$. 
Lemma 4.3. As $n \rightarrow \infty$, for $j=1, \ldots, \tilde{n}, j \neq q_{0}$,

$$
E\left(Z_{j, n}^{(i)}\right)^{2} \leq \begin{cases}C\left|j-q_{0}\right|, & i=1,2, \\ C\left|j-q_{0}\right|^{2 p}, & i=3,\end{cases}
$$

for any $p>1 / 3$ where $C$ does not depend on $n$ and $j$.

Proof. Since $\xi_{j, n}^{(1)}=\xi_{j, n}^{(2)}+\xi_{j, n}^{(3)}$, then relation (4.8) for $i=1$ follows if it is valid for $i=2,3$, for any $p>1 / 3$. The proof of (4.8) in case $i=2$ is the same as that of (4.9) in Robinson (1995b). Relation (4.8) in case $i=3$ follows since

$$
E\left(Z_{j, n}^{(3)}\right)^{2} \leq C\left|j-q_{0}\right|^{2 / 3}\left(\log \left|j-q_{0}\right|\right)^{4 / 3}
$$

which can be obtained similarly to relation (4.8) in Robinson (1995b), applying Lemma 4.1 and

$$
\frac{1}{2 \pi n} \int_{-\pi}^{\pi}\left|\frac{\phi(\lambda)}{\phi\left(\lambda_{j}\right)}-1\right|^{2}\left|\sum_{l=1}^{n} e^{i l\left(\lambda-\lambda_{j}\right)}\right|^{2} d \lambda \leq C\left|j-q_{0}\right|^{-1}
$$

which holds uniformly over $0 \leq j \leq \tilde{n}, j \neq q_{0}$. Estimate (4.10) generalizes Robinson's (1995b) Lemma 3 for all $\omega_{0} \in[0, \pi]$ and extends it over all Fourier frequences $\lambda_{j}, j \neq q_{0}$. The proof of (4.10) requires Assumption A.6. We omit the proof since, in general, it repeats the proof of Robinson's (1995b) Lemma 3. [Note that relation (4.8) in Robinson (1995b) where the peridogram $I_{j}$ is normalized by the approximate spectral density $C\left|\lambda_{j}-\lambda_{q_{0}}\right|^{-\alpha_{0}}, C>0$, due to the approximation, contains the additional term $\left|j-q_{0}\right|^{\beta+1} n^{-\beta}$.]

We now consider jointly covariance stationary processes $\left\{y_{t}\right\}$ and $\left\{z_{t}\right\}$, individually satisfying Assumptions A, (or more precisely, Assumptions A.5 and A.6) with the same innovations sequence $\left\{\varepsilon_{t}\right\}$, but with possibly different memory parameters, denoted $\alpha_{y}, \alpha_{z}$. In fact we allow also for $\alpha_{y}=0$ and/or $\alpha_{z}=0$, in order to apply Lemma 4.4 in the proof of Lemma 4.3 with $\left(y_{t}, z_{t}\right)$ representing $\left(x_{t}, x_{t}\right),\left(x_{t}, \varepsilon_{t}\right)$ and $\left(\varepsilon_{t}, \varepsilon_{t}\right)$. Denote by $f_{y}$ and $f_{z}$ the spectral densities of $y_{t}, z_{t}$, respectively, and by $R_{y z}(\lambda)$ their coherency. Introduce

$$
v_{y}(\lambda)=\left(\sum_{t=1}^{n} y_{t} e^{i t \lambda}\right) /\left\{f_{y}(\lambda) 2 \pi n\right\}^{1 / 2}, \quad v_{z}(\lambda)=\left(\sum_{t=1}^{n} z_{t} e^{i t \lambda}\right) /\left\{f_{z}(\lambda) 2 \pi n\right\}^{1 / 2} .
$$

LEMMA 4.4. Let the sequences $\left\{y_{t}\right\},\left\{z_{t}\right\}$ satisfy Assumptions A with $0 \leq$ $\alpha_{y}, \alpha_{z}<1$. Then, as $n \rightarrow \infty$, the following relations hold uniformly over $q_{0}+1 \leq k, j \leq \tilde{n}$ and $0 \leq k, j \leq q_{0}-1$ such that $\left|q_{0}-j\right|>\left|k-q_{0}\right|$ :

$$
\begin{gathered}
E\left[v_{y}\left(\lambda_{j}\right) \overline{v_{z}\left(\lambda_{j}\right)}\right]=R_{y z}\left(\lambda_{j}\right)+O\left(\left|j-q_{0}\right|^{-1} \log \left|j-q_{0}\right|\right) ; \\
E\left[v_{y}\left(\lambda_{j}\right) v_{z}\left(\lambda_{j}\right)\right]=O\left(\left|j-q_{0}\right|^{-1} \log \left|j-q_{0}\right|\right) ; \\
\max \left(\left|E\left[v_{y}\left(\lambda_{j}\right) \overline{v_{z}\left(\lambda_{k}\right)}\right]\right|,\left|E\left[v_{y}\left(\lambda_{j}\right) v_{z}\left(\lambda_{k}\right)\right]\right|\right)=O\left(\left|k-q_{0}\right|^{-1} \log \left|j-q_{0}\right|\right) .
\end{gathered}
$$


PROOF. We can write $y_{t}=\sum_{j=0}^{\infty} \phi_{y j} \varepsilon_{t-j}, z_{t}=\sum_{j=0}^{\infty} \phi_{z j} \varepsilon_{t-j}$, where $\left\{\phi_{y j}\right\}$, $\left\{\phi_{z j}\right\}$ satisfy the conditions on $\left\{\phi_{j}\right\}$ implied by Assumptions A. Further, $f_{y}$ and $f_{z}$ have representations of the form (4.1), with $\alpha$ replaced by $\alpha_{y} \in[0,1)$ and $\alpha_{z} \in[0,1)$, respectively, while the cross-spectral density of $\left(y_{t}, z_{t}\right)$ is of the form $\left(\sigma_{0}^{2} / 2 \pi\right) \phi_{y}(\lambda) \overline{\phi_{z}(\lambda)}$, where $\phi_{y}(\lambda)=\sum_{j=0}^{\infty} \phi_{y j} e^{i j \lambda}, \phi_{z}(\lambda)=\sum_{j=0}^{\infty} \phi_{z j} e^{i j \lambda}$, and thus has derivative in $\lambda$ that is $O\left(\left|\lambda-\omega_{0}\right|^{-1-\left(\alpha_{y}+\alpha_{z}\right) / 2}\right)$ for $\lambda \in[0, \pi] \backslash\left\{\omega_{0}\right\}$. It follows that Assumptions A.1-A.2 of Robinson (1995a) are satisfied, and the proof follows as in Robinson's (1995a) Theorem 2.

5. Proof of Lemma 4.1. Write, in the notation of (4.6),

$$
S_{n} \equiv \sum_{j\left(q_{0}\right)}{ }^{\prime} b_{j} \xi_{j, n}^{(1)}=\sum_{j\left(q_{0}\right)}{ }^{\prime} b_{j} \xi_{j, n}^{(2)}+\sum_{j\left(q_{0}\right)}{ }^{\prime} b_{j} \xi_{j, n}^{(3)}=: M_{n}+R_{n}
$$

(4.4) follows if we show that as $n \rightarrow \infty$,

$$
M_{n} \stackrel{d}{\Rightarrow} N\left(0, \sigma^{2}\right)
$$

and

$$
R_{n} \stackrel{P}{\Rightarrow} 0 .
$$

We begin with the proof of (5.2). By summation by parts,

$$
\begin{aligned}
E\left|R_{n}\right| & =E\left|\sum_{j=-1: j \neq q_{0}, q_{0}-1}^{\tilde{n}}\left(b_{j}-b_{j+1}\right) Z_{j, n}^{(3)}\right| \\
& \leq \sum_{j=-1: j \neq q_{0}, q_{0}-1}^{\tilde{n}}\left|b_{j}-b_{j+1}\right|\left(E\left(Z_{j, n}^{(3)}\right)^{2}\right)^{1 / 2} \leq C n_{3}(b, \gamma),
\end{aligned}
$$

by (4.8) where $\gamma>1 / 3$ is the same as in (4.3). So (5.2) follows by (4.3).

It remains to show (5.1). Since we employ a similar approach to that of Theorem 2 of Robinson (1995b) and $S_{n}$ is invariant to $\sigma_{0}^{2}$, we set $\sigma_{0}^{2}=1$ in the proof as he did. Write $M_{n}$ as

$$
M_{n}=\sum_{j=0}^{\tilde{n}} b_{j}\left(2 \pi I_{j, \varepsilon}-1\right)=\sum_{t=1}^{n} z_{t, n}
$$

where we can set $b_{q_{0}}=0$ because $M_{n}$ excludes $j=q_{0}$, and

$$
z_{t, n}=2 \varepsilon_{t} \sum_{s=1}^{t-1} c_{t-s} \varepsilon_{s}+c_{0}\left(\varepsilon_{t}^{2}-1\right), \quad c_{s}=n^{-1} \sum_{j=0}^{\tilde{n}} b_{j} \cos \left(s \lambda_{j}\right) .
$$

$z_{t, n}$ is a triangular martingale difference array since $E\left[z_{t, n} \mid F_{t-1}\right]=0$ by A.5. Thus, as in the proof of Theorem 2 of Robinson (1995b), (5.1) follows if

$$
V_{n}=\sum_{t=1}^{n} E\left(z_{t, n}^{2} \mid F_{t-1}\right) \stackrel{P}{\rightarrow} \sigma^{2} \quad(n \rightarrow \infty)
$$


and

$$
\sum_{t=1}^{n} E\left(z_{t, n}^{2} 1_{\left\{\left|z_{t, n}\right|>\delta\right\}}\right) \stackrel{P}{\rightarrow} 0 \quad \text { for all } \delta>0 .
$$

To derive (5.4) it suffices to show that

$$
E V_{n}=n_{2}(b)\left(1+n^{-2}\right)+\kappa_{4} n_{1}^{2}(b)
$$

and

$$
\operatorname{Var}\left(V_{n}\right) \rightarrow 0 \quad(n \rightarrow \infty)
$$

since together with (4.3) they imply (5.4). By definition of $z_{t, n}$,

$$
z_{t, n}^{2}=4 \varepsilon_{t}^{2} \sum_{1 \leq r, s<t} c_{t-s} c_{t-r} \varepsilon_{s} \varepsilon_{r}+c_{0}^{2}\left(\varepsilon_{t}^{2}-1\right)^{2}+4 \varepsilon_{t}\left(\varepsilon_{t}^{2}-1\right) c_{0} \sum_{1 \leq s<t} c_{t-s} \varepsilon_{s} .
$$

By Assumption A.5, $E\left[\varepsilon_{t}^{2} \mid F_{t-1}\right]=1, E\left[\left(\varepsilon_{t}^{2}-1\right)^{2} \mid F_{t-1}\right]=\mu_{4}-1, E\left[\varepsilon_{t}\left(\varepsilon_{t}^{2}-\right.\right.$ 1) $\left.\mid F_{t-1}\right]=\mu_{3}$. Therefore

$$
V_{n}=4 \sum_{t=1}^{n} \sum_{1 \leq r, s<t} c_{t-s} c_{t-r} \varepsilon_{s} \varepsilon_{r}+\left(\mu_{4}-1\right) c_{0}^{2} n+4 \mu_{3} c_{0} \sum_{t=1}^{n} \sum_{1 \leq s<t} c_{t-s} \varepsilon_{s},
$$

and thus

$$
E V_{n}=4 \sum_{t=1}^{n} \sum_{s=1}^{t-1} c_{t-s}^{2}+\left(\mu_{4}-1\right) c_{0}^{2} n
$$

Since by definition

$$
n c_{0}^{2}=n_{1}^{2}(b)
$$

(5.6) follows from (5.8) if

$$
i_{n}:=4 \sum_{t=2}^{n} \sum_{s=1}^{t-1} c_{t-s}^{2}=n_{2}(b)\left(1+n^{-2}\right)-2 n_{1}^{2}(b)
$$

which can be obtained using similar algebra as in Robinson (1995b). Indeed,

$$
\begin{aligned}
i_{n}= & 4 \sum_{t=1}^{n-1} \sum_{s=1}^{n-t} c_{s}^{2}=4 n^{-2} \sum_{j, k=0}^{\tilde{n}} b_{j} b_{k} \sum_{t=1}^{n-1} \sum_{s=1}^{n-t}\left[\cos \left(s \lambda_{j}\right) \cos \left(s \lambda_{k}\right)\right] \\
= & 4 n^{-2} \sum_{j=0}^{n} b_{j}^{2} \sum_{t=1}^{n-1} \sum_{s=1}^{n-t} \cos ^{2}\left(s \lambda_{j}\right) \\
& +2 n^{-2} \sum_{j, k=0: j \neq k}^{n} b_{j} b_{k} \sum_{t=1}^{n-1} \sum_{s=1}^{n-t}\left[\cos \left(s\left(\lambda_{j}+\lambda_{k}\right)\right)+\cos \left(s\left(\lambda_{j}-\lambda_{k}\right)\right)\right],
\end{aligned}
$$

and applying the equalities

$$
\sum_{t=1}^{n-1} \sum_{s=1}^{n-t} \cos ^{2}\left(s \lambda_{j}\right)=(n-1)^{2} / 4, \quad \sum_{t=1}^{n-1} \sum_{s=1}^{n-t}\left[\cos \left(s\left(\lambda_{j}+\lambda_{k}\right)\right)+\cos \left(s\left(\lambda_{j}-\lambda_{k}\right)\right)\right]=-n
$$


we obtain

$$
\begin{aligned}
i_{n} & =n^{-2}(n-1)^{2} \sum_{j=0}^{\tilde{n}} b_{j}{ }^{2}-2 n^{-1} \sum_{j, k=0: j \neq k}^{\tilde{n}} b_{j} b_{k} \\
& =\sum_{j=0}^{\tilde{n}} b_{j}{ }^{2}\left(1+n^{-2}-2 n^{-1}\right)-2 n^{-1}\left[\left(\sum_{j=0}^{\tilde{n}} b_{j}\right)^{2}-\sum_{j=0}^{\tilde{n}} b_{j}{ }^{2}\right] \\
& =n_{2}(b)\left(1+n^{-2}\right)-2 n_{1}^{2}(b),
\end{aligned}
$$

establishing (5.6).

Next we show (5.7). We have

$$
\begin{aligned}
V_{n}-E V_{n}= & 4 \sum_{t=1}^{n} \sum_{1 \leq r, s<t} c_{t-s} c_{t-r}\left(\varepsilon_{s} \varepsilon_{r}-E\left[\varepsilon_{s} \varepsilon_{r}\right]\right) \\
& +4 \mu_{3} c_{0} \sum_{t=1}^{n} \sum_{1 \leq s<t} c_{t-s} \varepsilon_{s} .
\end{aligned}
$$

By (5.11) and elementary inequalities,

$$
\begin{aligned}
\operatorname{Var}\left(V_{n}\right) \leq & 32\left(E\left(\sum_{t=1}^{n} \sum_{1 \leq r, s<t} c_{t-s} c_{t-r}\left(\varepsilon_{s} \varepsilon_{r}-E\left[\varepsilon_{s} \varepsilon_{r}\right]\right)\right)^{2}\right. \\
& \left.+E\left(\mu_{3}^{2} c_{0}^{2}\left(\sum_{t=1}^{n} \sum_{1 \leq s<t} \varepsilon_{s} c_{t-s}\right)^{2}\right)\right) \\
\leq & C\left(\sum_{t_{1}, t_{2}=1}^{n}\left[\sum_{s=1}^{\min \left(t_{1}, t_{2}\right)}\left|c_{t_{1}-s} c_{t_{2}-s}\right|\right]^{2}+c_{0}^{2} \sum_{t_{1}, t_{2}=1}^{n}\left[\sum_{s=1}^{\min \left(t_{1}, t_{2}\right)}\left|c_{t_{1}-s} c_{t_{2}-s}\right|\right]\right) .
\end{aligned}
$$

For $1 \leq s \leq n-1,\left|c_{s}\right|=o(1) n^{-p} \min (s, n-s)^{p-1}$ by Lemma 5.1 below and (4.3) for any $p \in(1 / 3, \min (\gamma, 1 / 2))$. Thus,

$$
\begin{aligned}
& \sum_{s=1}^{\min \left(t_{1}, t_{2}\right)}\left|c_{t_{1}-s} c_{t_{2}-s}\right| \\
& =o\left(n^{-2 p}\right) \sum_{s=1}^{\min \left(t_{1}, t_{2}\right)}\left\{\min \left(\left|t_{1}-s\right|_{+},\left|t_{1}-s-n\right|_{+}\right)^{p-1}\right. \\
& \left.\quad \times \min \left(\left|t_{2}-s\right|_{+},\left|t_{2}-s-n\right|_{+}\right)^{p-1}\right\} \\
& =o\left(n^{-2 p}\right) \min \left(\left|t_{1}-t_{2}\right|_{+},\left|t_{1}-t_{2}-n\right|_{+}\right)^{2 p-1}
\end{aligned}
$$

uniformly in $t_{1}, t_{2}$, where $|t|_{+}=\max (|t|, 1)$. By (5.9) and (4.3), $c_{0}^{2}=O\left(n^{-1}\right)$. Hence,

$$
\begin{aligned}
\operatorname{Var}\left(V_{n}\right) & =o(1)\left(n^{-4 p} \sum_{t_{1}, t_{2}=1}^{n}\left|t_{1}-t_{2}\right|_{+}^{2(2 p-1)}+n^{-2 p-1} \sum_{t_{1}, t_{2}=1}^{n}\left|t_{1}-t_{2}\right|_{+}^{2 p-1}\right) \\
& =o(1)\left(n^{-4 p} \sum_{t_{1}=1}^{n} \sum_{u=-n}^{n}|u|_{+}^{4 p-2}+n^{-2 p-1} \sum_{t_{1}=1}^{n} \sum_{u=-n}^{n}|u|_{+}^{2 p-1}\right)=o(1)
\end{aligned}
$$

since $p>1 / 3$. Thus, (5.7) is established, to complete the proof of (5.4). 
To complete the proof of Lemma 4.1 we need to examine (5.5). Write

$$
z_{t, n}=2\left\{\varepsilon_{t} \sum_{1 \leq s<t} \varepsilon_{s} c_{t-s}\right\}+\left\{c_{0}\left(\varepsilon_{t}^{2}-1\right)\right\}=: X_{t}+Y_{t} .
$$

Then

$$
\begin{aligned}
& E\left[z_{t, n}^{2} 1_{\left\{\left|z_{t, n}\right| \geq \delta\right\}}\right] \leq E\left[\left(\left|X_{t}\right|+\left|Y_{t}\right|\right)^{2} 1_{\left\{\left|X_{t}+Y_{t}\right| \geq \delta\right\}}\right] \\
& \quad \leq E\left[\left(2\left|X_{t}\right|\right)^{2} 1_{\left\{\left|X_{t}\right| \geq\left|Y_{t}\right|,\left|X_{t}\right| \geq \delta / 2\right\}}\right]+E\left[\left(2\left|Y_{t}\right|\right)^{2} 1_{\left\{\left|Y_{t}\right| \geq\left|X_{t}\right|,\left|Y_{t}\right| \geq \delta / 2\right\}}\right] .
\end{aligned}
$$

The first term in (5.12) is bounded by

$$
\begin{aligned}
4 E\left[\left|X_{t}\right|^{2} 1_{\left\{\left|X_{t}\right| \geq \delta / 2\right\}}\right] & \leq 4(\delta / 2)^{-2} E\left|X_{t}\right|^{4}=C E\left(\varepsilon_{t}\right)^{4} E\left(\sum_{1 \leq s<t} \varepsilon_{s} c_{t-s}\right)^{4} \\
& \leq C\left(\sum_{s=1}^{t-1} c_{s}^{2}\right)^{2} \leq C n^{-4 p}\left(\sum_{s=1}^{\infty} s^{2 p-2}\right)^{2} \leq C n^{-4 p}
\end{aligned}
$$

applying (5.13) with $p \in(1 / 3, \min (\gamma, 1 / 2))$. The second term of (5.12) is bounded by

$$
\begin{aligned}
4 E\left[\left|Y_{t}\right|^{2} 1_{\left\{\left|Y_{t}\right| \geq \delta / 2\right\}}\right] & \leq 4(\delta / 2)^{-\eta / 2} E\left|Y_{t}\right|^{2+\eta / 2} \leq C E\left|c_{0}\left(\varepsilon_{t}^{2}-1\right)\right|^{2+\eta / 2} \\
& \leq C\left|c_{0}\right|^{2+\eta / 2} E\left|\varepsilon_{t}\right|^{4+\eta}=o\left(n^{-1}\right)
\end{aligned}
$$

since $\left|c_{0}\right| \leq C n^{-1 / 2}$ and by Assumption A.5, $E\left|\varepsilon_{t}\right|^{4+\eta}<\infty$ for some $\eta>0$. Hence

$$
\sum_{t=1}^{n} E\left[z_{t, n}^{2} 1_{\left\{\left|z_{t, n}\right| \geq \delta\right\}}\right]=\left[O\left(n^{-4 p}\right)+o\left(n^{-1}\right)\right] \sum_{t=1}^{n} 1=o(1) \quad(n \rightarrow \infty) .
$$

This completes the proof of (5.5) and Lemma 4.1.

LEMMA 5.1. Under the assumptions of Lemma 4.1, for any $0<p<1$,

$$
\left|c_{s}\right| \leq C n^{-p} \min (s, n-s)^{p-1} n_{3}(b, p)
$$

uniformly in $1 \leq s \leq n-1$.

PROOF. Recall that $b_{\tilde{n}+1}=b_{-1}=0$. Summation by parts implies

$$
c_{s}=n^{-1}\left\{\sum_{j=q_{0}+1}^{\tilde{n}}\left(b_{j}-b_{j+1}\right) \sum_{l=q_{0}+1}^{j} \cos \left(l \lambda_{s}\right)+\sum_{j=0}^{q_{0}-1}\left(b_{j}-b_{j-1}\right) \sum_{l=j}^{q_{0}-1} \cos \left(l \lambda_{s}\right)\right\} .
$$

Note that $\left|\sum_{l=k+1}^{m} \cos \left(l \lambda_{s}\right)\right| \leq|m-k|^{p}\left|\sum_{l=k+1}^{m} \cos \left(l \lambda_{s}\right)\right|^{1-p} \leq C|m-k|^{p}$ $(n / \min (s, n-s))^{1-p}$ which holds because $\left|\sum_{l=k+1}^{m} \cos \left(l \lambda_{s}\right)\right| \leq C\left|\sin \left(\lambda_{s} / 2\right)\right|^{-1} \leq$ 
$C(n / \min (s, n-s))$ uniformly in $0 \leq k<m$ and $1 \leq s \leq n-1$. Thus

$$
\begin{aligned}
&\left|c_{s}\right| \leq(n / \min (s, n-s))^{1-p} n^{-1}\left[\sum_{j=q_{0}+1}^{\tilde{n}}\left|b_{j}-b_{j+1}\right|\left|j-q_{0}\right|^{p}\right. \\
&\left.+\sum_{j=0}^{q_{0}-1}\left|b_{j}-b_{j-1}\right|\left|j-q_{0}\right|^{p}\right] \\
& \equiv C n^{-p} \min (s, n-s)^{p-1} n_{3}(b, p) .
\end{aligned}
$$

LEMMA 5.2. Under the conditions of Theorem 3.2 the convergence (3.22) holds.

Proof. By Theorem 3.1, it suffices to consider $\widehat{\omega}=2 \pi \hat{q} / n$ such that $\mid \hat{q}-$ $q_{0} \mid \leq K$ where $K>0$ is large enough. The $\ell$ th element of $n^{1 / 2} \nabla_{\theta} S\left(\theta_{0}, \lambda_{q}\right)$ equals to $(n / \tilde{n}) W_{n, q}^{(\ell)}$, where

$$
W_{n, q}^{(\ell)}=n^{-1 / 2} \sum_{j=0}^{\tilde{n}}\left(\partial / \partial \theta_{\ell}\right) k_{j}^{-1}\left(\theta_{0}, \lambda_{q}\right) I_{j}
$$

and $\theta_{\ell}$ is the $\ell$ th element of $\theta$. Setting $h_{n, q}^{(\ell)}(\lambda)=\left(\sigma_{0}^{2} / 2 \pi\right) k\left(\lambda ; \psi_{0}\right)\left(\partial / \partial \theta_{\ell}\right) k^{-1}$ $\left(\lambda, \theta_{0}, \lambda_{q}\right)$, write $W_{n, q}^{(\ell)}=v_{n, q}^{(\ell)}+e_{n, q}^{(\ell)}+r_{n, q}^{(\ell)}$ where

$$
v_{n, q}^{(\ell)}=n^{-1 / 2} \sum_{j\left(q_{0}\right)}^{\prime} h_{n, q}^{(\ell)}\left(\lambda_{j}\right)\left(\frac{2 \pi I_{j}}{\sigma_{0}^{2} k_{j}\left(\psi_{0}\right)}-1\right), \quad e_{n, q}^{(\ell)}=n^{-1 / 2} \sum_{j\left(q_{0}\right)}^{\prime} h_{n, q}^{(\ell)}\left(\lambda_{j}\right)
$$

and $r_{n, q}^{(\ell)}=n^{-1 / 2}\left(\partial / \partial \theta_{\ell}\right) k_{q_{0}}^{-1}\left(\theta_{0}, \lambda_{q}\right) I_{q_{0}}$. To complete the proof it suffices to show: (i) that as $n \rightarrow \infty$,

$$
\left(v_{n, q_{0}}^{(\ell)}\right)_{\ell=1, \ldots, p} \stackrel{d}{\Rightarrow}\left(Z^{(\ell)}\right)_{\ell=1, \ldots, p},
$$

where $\left(Z^{(1)}, \ldots, Z^{(p)}\right)^{\prime}$ is a Gaussian vector with zero mean and covariance $E\left(Z^{(\ell)} Z^{\left(\ell^{\prime}\right)}\right)=(2 \pi)^{-2} \sigma_{0}^{4} w_{\ell \ell^{\prime}}$; and (ii) that for $k=-K, \ldots, K ; \ell=1, \ldots, p$,

$$
\begin{gathered}
v_{n, q_{0}+k}^{(\ell)}-v_{n, q_{0}}^{(\ell)} \stackrel{P}{\Rightarrow} 0, \\
e_{n, q_{0}+k}^{(\ell)} \rightarrow 0, \quad E\left|r_{n, q_{0}+k}^{(\ell)}\right| \rightarrow 0 .
\end{gathered}
$$

(5.14)-(5.16) imply (3.22). Also, as a by-product, (5.14)-(5.15) imply that the distribution of $\hat{\theta}$ in Theorem 3.2 is the same whether $\omega_{0}$ is known or estimated.

By the Cramér-Wold device, the convergence (5.14) holds if for any sequence of real numbers $a_{1}, \ldots, a_{p}, p \geq 1$, as $n \rightarrow \infty$,

$$
Q_{n}:=\sum_{\ell=1}^{p} a_{\ell} v_{n, q_{0}}^{(\ell)} \stackrel{d}{\Rightarrow} Q:=\sum_{\ell=1}^{p} a_{\ell} Z^{(\ell)}
$$


Write

$$
Q_{n}=n^{-1 / 2} \sum_{j\left(q_{0}\right)}^{\prime} h_{n}\left(\lambda_{j}\right)\left(2 \pi\left(\sigma_{0}^{2} k_{j}\left(\psi_{0}\right)\right)^{-1} I_{j}-1\right), \quad h_{n}(\lambda)=\sum_{\ell=1}^{p} a_{\ell} h_{n, q_{0}}^{(\ell)}(\lambda) .
$$

To derive (5.17) we apply Theorem 4.2. Note that $\lambda_{q_{0}} \rightarrow \omega_{0}$ as $n \rightarrow \infty$, so that

$$
h_{n}(\lambda) \rightarrow h(\lambda)=\sum_{\ell=1}^{p} a_{\ell} h^{(\ell)}(\lambda), \quad h^{(\ell)}(\lambda)=-\left(\sigma_{0}^{2} / 2 \pi\right)\left(\partial / \partial \theta_{\ell}\right) \log k\left(\lambda ; \theta_{0}, \omega_{0}\right) .
$$

By Assumption A.2, $\int_{0}^{\pi} h^{(\ell)}(\lambda) d \lambda=0, \int_{0}^{\pi} h(\lambda) d \lambda=0$. In addition, from Assumption A. $1^{\prime}$ or A.1 it is easy to obtain that there exists $\varepsilon \in(0,1 / 2)$ such that

$$
\begin{gathered}
\left|h_{n, q_{0}}^{(\ell)}(\lambda)\right| \leq C\left|\lambda-\omega_{0}\right|^{-\varepsilon}, \\
\left|(d / d \lambda) h_{n, q_{0}}^{(\ell)}(\lambda)\right| \leq C\left|\lambda-\omega_{0}\right|^{-1-\varepsilon}
\end{gathered}
$$

uniformly in $\left|\lambda-\omega_{0}\right| \geq 2 K / n$. Therefore, the functions $h_{n}, n \geq 1$ satisfy conditions (c1)-(c3) of Theorem 4.2 which yields $Q_{n} \stackrel{d}{\Rightarrow} Q=N\left(0, s^{2}\right), s^{2}=$ $(2 \pi)^{-1} \int_{0}^{\pi} h^{2}(\lambda) d \lambda$. Hence (5.14) holds.

To show (5.15), write

$$
v_{n, q_{0}+k}^{(\ell)}-v_{n, q_{0}}^{(\ell)}=n^{-1 / 2} \sum_{j\left(q_{0}\right)}^{\prime} \tilde{h}_{n}\left(\lambda_{j}\right)\left(\left(2 \pi / \sigma_{0}^{2}\right) k_{j}\left(\psi_{0}\right)^{-1} I_{j}-1\right)
$$

where for $\lambda \neq \omega_{0}, \tilde{h}_{n}(\lambda)=h_{n, q_{0}+k}^{(\ell)}(\lambda)-h_{n, q_{0}}^{(\ell)}(\lambda) \rightarrow 0$ as $n \rightarrow \infty$. Then the convergence (5.15) follows by the same argument as in the proof of (5.14).

Finally we show (5.16). By (5.18)-(5.19) and Lemma 5.3 below,

$$
e_{n, q_{0}+k}^{(\ell)}=\left(n^{1 / 2} / 2 \pi\right) \int_{0}^{\pi} 1_{\left\{\left|\lambda-\omega_{0}\right| \geq 2 K / n\right\}} h_{n, q_{0}+k}^{(\ell)}(\lambda) d \lambda+o(1) .
$$

Furthermore, since $\int_{0}^{\pi} h^{(\ell)}(\lambda) d \lambda=0$,

$$
\begin{aligned}
\left|e_{n, q_{0}+k}^{(\ell)}\right| \leq & C n^{1 / 2} \int_{0}^{\pi} 1_{\left\{\left|\lambda-\omega_{0}\right| \geq 2 K / n\right\}}\left|h_{n, q_{0}+k}^{(\ell)}(\lambda)-h^{(\ell)}(\lambda)\right| d \lambda \\
& +C n^{1 / 2} \int_{0}^{\pi} 1_{\left\{\left|\lambda-\omega_{0}\right|<2 K / n\right\}}\left|h^{(\ell)}(\lambda)\right| d \lambda+o(1) .
\end{aligned}
$$

It is easy to check that under Assumptions A (i.e., under Assumptions A.1 or A. $\left.1^{\prime}\right)$, uniformly in $\left|\lambda-\omega_{0}\right| \geq 2 K / n$,

$$
\begin{aligned}
& \left|h_{n, q_{0}+k}^{(\ell)}(\lambda)-h^{(\ell)}(\lambda)\right| \\
& \quad=\frac{\sigma_{0}^{2}}{2 \pi}\left|\frac{k\left(\lambda ; \theta_{0}, \lambda_{q_{0}}\right)}{k\left(\lambda ; \theta_{0}, \lambda_{q_{0}+k}\right)} \frac{\partial}{\partial \theta_{\ell}} \log k\left(\lambda ; \theta_{0}, \lambda_{q_{0}+k}\right)-\frac{\partial}{\partial \theta_{\ell}} \log k\left(\lambda ; \theta_{0}, \omega_{0}\right)\right| \\
& \quad \leq C\left(\lambda_{k}\left(\lambda-\lambda_{q_{0}}\right)^{-1}+\lambda_{k}^{\beta}\right) \log n \leq C\left(n^{-1}\left(\lambda-\lambda_{q_{0}}\right)^{-1}+n^{-\beta}\right) \log n
\end{aligned}
$$


where $\beta \in(1 / 2,1)$ is the Lipschitz parameter in Assumption A.1' (or A.1), and $\left|h^{(\ell)}(\lambda)\right|=\left|\left(\partial / \partial \theta_{\ell}\right) \log k\left(\lambda ; \theta_{0}, \lambda_{q_{0}+k}\right)\right| \leq C\left|\lambda-\lambda_{q_{0}}\right|^{-\varepsilon}$ with $0<\varepsilon<1 / 2$. Thus, as $n \rightarrow \infty$,

$$
\begin{aligned}
\left|e_{n, q_{0}+k}^{(\ell)}\right| \leq & C n^{1 / 2}\left(\log n \int_{0:\left|\lambda-\omega_{0}\right| \geq 2 K / n}^{\pi}\left(n^{-1}\left(\lambda-\lambda_{q_{0}}\right)^{-1}+n^{-\beta}\right) d \lambda\right. \\
& \left.+C n^{1 / 2} \int_{0:\left|\lambda-\omega_{0}\right|<2 K / n}^{\pi}\left|\lambda-\lambda_{q_{0}}\right|^{-\varepsilon} d \lambda\right)+o(1) \\
& =o(1) .
\end{aligned}
$$

It remains to estimate $E\left|r_{n, q_{0}+k}^{(\ell)}\right|$. Since $\left|\left(\partial / \partial \theta_{\ell}\right) k^{-1}\left(\lambda_{q_{0}} ; \theta_{0}, \lambda_{q_{0}+k}\right)\right| \leq C\left|\lambda_{k}\right|^{\alpha_{0}}$ $\left|\log \lambda_{k}\right| \leq C n^{-\alpha_{0}} \log n$, then $E\left|r_{n, q_{0}+k}^{(\ell)}\right| \leq C n^{-1 / 2-\alpha_{0}} \log n E I_{q_{0}} \rightarrow 0$ follows by standard arguments.

The following lemma is used in the proofs of Lemma 5.2 and Lemma 7.1, Section 7.

LEMMA 5.3. Suppose the functions $\psi_{n}(\lambda), n \geq 1$, satisfy Assumptions (c1)(c3) of Theorem 4.2 on $h_{n}(\lambda)$ with $0 \leq \alpha^{\prime}<1$ and $0 \leq \gamma^{\prime}<2, \gamma^{\prime} \neq 1$. Then, for large enough $K>0$,

$$
\begin{aligned}
n^{-1} \sum_{j\left(q_{0}\right)}{ }^{\prime} \psi_{n}\left(\lambda_{j}\right)= & (2 \pi)^{-1} \int_{0}^{\pi} 1_{\left\{\left|\lambda-\omega_{0}\right| \geq K / n\right\}} \psi_{n}(\lambda) d \lambda \\
& +O\left(n^{-1+\max \left(\alpha^{\prime}, \gamma^{\prime}-1\right)}\right) \\
= & (2 \pi)^{-1} \int_{0}^{\pi} \psi(\lambda) d \lambda+o(1) .
\end{aligned}
$$

Proof. Denote by $J_{n}$ the left hand side of (5.20). By (c1),

$$
\begin{aligned}
J_{n}= & n^{-1} \sum_{j=0:\left|j-q_{0}\right| \geq K}^{\tilde{n}-1} \psi_{n}\left(\lambda_{j}\right)+O\left(n^{\alpha^{\prime}-1}\right) \\
= & n^{-1} \sum_{j=0:\left|j-q_{0}\right| \geq K}^{n-1} \int_{j}^{j+1}\left(\psi_{n}(2 \pi[\lambda] / n)-\psi_{n}(2 \pi \lambda / n)\right) d \lambda \\
& +n^{-1} \int_{0}^{\tilde{n}} 1_{\left\{\left|\lambda-q_{0}\right| \geq K\right\}} \psi_{n}(2 \pi \lambda / n) d \lambda+O\left(n^{\alpha^{\prime}-1}\right)=: i_{n, 1}+i_{n, 2} .
\end{aligned}
$$

By (4.5)

$$
i_{n, 1}=O\left(n^{-2} \sum_{j=0:\left|j-q_{0}\right| \geq K}^{\tilde{n}}\left(\left|j-q_{0}\right| / n\right)^{-\gamma^{\prime}}\right)=O\left(n^{-1+\max \left(0, \gamma^{\prime}-1\right)}\right),
$$

whereas by (c1), (c3) and Lebesgue's dominated convergence theorem,

$$
i_{n, 2}=(2 \pi)^{-1} \int_{0:\left|\lambda-\omega_{0}\right| \geq 2 \pi K / n}^{\pi} \psi_{n}(\lambda) d \lambda=(2 \pi)^{-1} \int_{0}^{\pi} \psi(\lambda) d \lambda+o(1) .
$$

(5.21)-(5.23) imply (5.20). 
The following Lemma 5.4 is used in the proof of Lemma 6.1.

LEMMA 5.4. Under the assumptions of Lemma 4.3 and with $Z_{j, n}^{(2)}$ defined by (4.7),

$$
\left|Z_{j, n}^{(2)}\right| \leq C\left|j-q_{0}\right|^{1 / 2} Z_{n}+\tilde{Z}_{j, n}^{(2)}
$$

where $Z_{n}=n^{-1 / 2}\left|\sum_{t=1}^{n}\left(\varepsilon_{t}^{2}-\sigma_{0}^{2}\right)\right|$ and $\tilde{Z}_{j, n}^{(2)}=2 \sigma_{0}^{-2}\left|\sum_{1 \leq s<t \leq n} c_{t-s} \varepsilon_{t} \varepsilon_{s}\right|$, which satisfy

$$
E Z_{n}^{2} \leq C, \quad E\left(\tilde{Z}_{j, n}^{(2)}\right)^{4} \leq C\left|j-q_{0}\right|^{2},
$$

uniformly in $j=0, \ldots, \tilde{n}\left(j \neq q_{0}\right)$.

Proof. Set $b_{l}=1_{\left\{q_{0}+1 \leq l \leq j\right\}}$ if $j>q_{0} ; b_{l}=1_{\left\{j+1 \leq l \leq q_{0}-1\right\}}$ if $j<q_{0}$. By (4.7), similarly to (5.3),

$$
Z_{j, n}^{(2)}=\sum_{l=0}^{\tilde{n}} b_{l} \xi_{l, n}^{(2)}=\sigma_{0}^{2} \sum_{t=1}^{n} z_{t, n} \quad \text { where } z_{t, n}=\frac{2 \varepsilon_{t}}{\sigma_{0}^{2}} \sum_{s=1}^{t-1} c_{t-s} \varepsilon_{s}+c_{0}\left(\frac{\varepsilon_{t}^{2}}{\sigma_{0}^{2}}-1\right) .
$$

Since $c_{0}=n^{-1 / 2} n_{1}(b)=n^{-1} \sum_{j\left(q_{0}\right)}^{\prime} b_{j}=n^{-1}\left|j-q_{0}\right| \leq n^{-1 / 2}\left|j-q_{0}\right|^{1 / 2}$, (5.24) follows. Clearly by A.5, $E Z_{n}^{2} \leq C$. On the other hand,

$$
E\left(\tilde{Z}_{j, n}^{(2)}\right)^{4}=16 \prod_{i=1}^{4} \sum_{1 \leq s_{i}<t_{i} \leq n} c_{t_{i}-s_{i}} E\left[\varepsilon_{t_{1}} \varepsilon_{s_{1}} \ldots \varepsilon_{t_{4}} \varepsilon_{s_{4}}\right] .
$$

Since the number of equal indices in the set $\left\{t_{1}, s_{1}, \cdots, t_{4}, s_{4}\right\}$ does not exceed 4, by Assumption A.5 it follows $\left|E\left[\varepsilon_{t_{1}} \varepsilon_{s_{1}} \ldots \varepsilon_{t_{4}} \varepsilon_{s_{4}}\right]\right| \leq C$. Moreover, by A.5, the inequality $E\left[\varepsilon_{t_{1}} \varepsilon_{s_{1}} \cdots \varepsilon_{t_{4}} \varepsilon_{s_{4}}\right] \neq 0$ can hold only if any $t_{i}, s_{i}$ are repeated in $\left\{t_{1}, s_{1}, \ldots, t_{4}, s_{4}\right\}$ at least twice. Hence, applying the Cauchy inequality we obtain that

$$
\begin{aligned}
E\left(\tilde{Z}_{j, n}^{(2)}\right)^{4} & \leq C \prod_{i=1}^{4}\left(\sum_{1 \leq s_{i}<t_{i} \leq n} c_{t_{i}-s_{i}}^{2}\right)^{1 / 2}=C\left(\sum_{1 \leq s<t \leq n} c_{t-s}^{2}\right)^{2} \\
& \leq C\left(n_{2}(b)+n_{1}(b)^{2}\right)^{2} \leq C n_{2}(b)^{2}=4\left|j-q_{0}\right|^{2},
\end{aligned}
$$

by (5.10), which proves (5.25).

\section{Proof of (3.17).}

LEMMA 6.1. Under the assumptions of Theorem 3.1, there exists $\eta>0$ such that as $n \rightarrow \infty$,

$$
E \sup _{\psi \in \Psi_{K}} u_{n}(\psi)^{-1}\left|U_{n}(\psi)\right| \leq C K^{-\eta}
$$

where $C<\infty$ does not depend on $n$ and $K$. 
PRoof. From (3.16) we may write $U_{n}(\psi)=\sum_{j\left(q_{0}\right)}^{\prime} b_{j}(\psi) \xi_{j, n}^{(1)}$ where

$$
b_{j}(\psi)=\frac{k_{j}\left(\psi_{0}\right)}{k_{j}(\psi)}-1, \quad \xi_{j, n}^{(1)}=\frac{2 \pi I_{j}}{\sigma_{0}^{2} k_{j}\left(\psi_{0}\right)}-1
$$

By summation by parts

$$
\left|U_{n}(\psi)\right| \leq \sum_{j=-1: j \neq q_{0}, q_{0}-1}^{\tilde{n}}\left|b_{j}(\psi)-b_{j+1}(\psi)\right|\left|Z_{j, n}^{(1)}\right|
$$

where $b_{-1}(\psi)=b_{\tilde{n}+1}(\psi)=0$ and $Z_{j, n}^{(1)}$ is defined by (4.7). Then $\left|U_{n}(\psi)\right| \leq$ $U_{n}^{(1)}(\psi)+U_{n}^{(2)}(\psi)$, where

$$
\begin{aligned}
& U_{n}^{(1)}(\psi)=\sum_{j=0: j \neq q_{0}, q_{0}-1}^{\tilde{n}-1}\left|b_{j}(\psi)-b_{j+1}(\psi)\right|\left|Z_{j, n}^{(1)}\right|, \\
& U_{n}^{(2)}(\psi)=1_{\left\{q_{0}<\tilde{n}\right\}}\left|b_{\tilde{n}}(\psi) Z_{\tilde{n}, n}^{(1)}\right|+1_{\left\{q_{0}>0\right\}}\left|b_{0}(\psi) Z_{-1, n}^{(1)}\right| .
\end{aligned}
$$

It suffices to show that

$$
E \sup _{\psi \in \Psi_{K}} u_{n}(\psi)^{-1}\left|U_{n}^{(i)}(\psi)\right| \leq C K^{-\eta}, \quad i=1,2 .
$$

We begin with $i=2$. We have

$$
\begin{aligned}
& E\left(\sup _{\psi \in \Psi_{K}} u_{n}(\psi)^{-1} U_{n}^{(2)}(\psi)\right) \\
& \quad \leq \sup _{\psi \in \Psi_{K}} u_{n}(\psi)^{-1}\left[1_{\left\{q_{0}<\tilde{n}\right\}}\left|b_{\tilde{n}}(\psi)\right|+1_{\left\{q_{0}>\tilde{n}\right\}}\left|b_{0}(\psi)\right|\right]\left(E\left|Z_{\tilde{n}, n}^{(1)}\right|+E\left|Z_{-1, n}^{(1)}\right|\right) \\
& \quad \leq C K^{-1 / 2}
\end{aligned}
$$

since, by (6.18) below, $1_{\left\{q_{0}<\tilde{n}\right\}}\left|b_{\tilde{n}}(\psi)\right|+1_{\left\{q_{0}>0\right\}}\left|b_{0}(\psi)\right| \leq C\left(|| \theta-\theta_{0} \|+\left|\lambda_{\bar{q}}\right|^{1 / 2}\right) \leq$ $C n^{-1 / 2} u_{n}(\psi)^{1 / 2}$ and, by Lemma $4.3, E\left|Z_{\tilde{n}, n}^{(1)}\right|+E\left|Z_{-1, n}^{(1)}\right| \leq C n^{1 / 2}$.

Next we show (6.2) for $i=1$. Set $A=\left\{\psi:\left\|\theta-\theta_{0}\right\| \leq(\log n)^{-1}\right\}$ and $B=\left\{\psi:\left\|\theta-\theta_{0}\right\|>(\log n)^{-1}\right\}$. Since by Lemma 6.3 below, $\left|b_{j}(\psi)-b_{j+1}(\psi)\right| \leq$ $C\left(\beta_{j, 1}(\psi)+\beta_{j, 2}(\psi)\right)$, where

$$
\begin{aligned}
\beta_{j, 1}(\psi)= & \left(\frac{|| \theta-\theta_{0}||+\left|\lambda_{\bar{q}}\right|^{1 / 2}}{\left|j-q_{0}\right|_{+}}+|\bar{q}|_{+}^{1-\varepsilon}\left|j-q_{0}\right|_{+}^{-(2-\varepsilon)}\right) 1_{A} \\
& +n^{1-\varepsilon}\left|j-q_{0}\right|_{+}^{-(2-\varepsilon)} 1_{B},
\end{aligned}
$$

$$
\beta_{j, 2}(\psi)=|\bar{q}|_{+}\left|j-q_{0}\right|_{+}^{-1}|j-q|_{+}^{-1} 1_{A}+n^{1-\varepsilon}\left|j-q_{0}\right|_{+}^{-(1-\varepsilon)}|j-q|_{+}^{-1} 1_{B}
$$


for $j=0, \ldots, \tilde{n}-1\left(j \neq q_{0}, q_{0}-1\right)$, where $0<\varepsilon<1 / 2$ is an arbitrarily small positive number, it follows that

$$
\begin{aligned}
& E\left(\sup _{\psi \in \Psi_{K}} u_{n}(\psi)^{-1}\left|U_{n}^{(1)}(\psi)\right|\right) \\
& \leq C\left(E \sum_{j=0: j \neq q_{0}, q_{0}-1}^{n \operatorname{nup}_{\psi \in \Psi_{K}}} u_{n}(\psi)^{-1} \beta_{j, 1}(\psi)\left|Z_{j, n}^{(1)}\right|\right. \\
&\left.+E \sum_{j=0: j \neq q_{0}, q_{0}-1} \sup _{k=0, \ldots, \tilde{n}} \sup _{\theta: \psi=\left(\theta, \lambda_{k}\right) \in \Psi_{K}} u_{n}(\psi)^{-1} \beta_{j, 2}(\psi)\left|Z_{j, n}^{(1)}\right|\right) \\
&= C\left(A_{n}+R_{n}\right) .
\end{aligned}
$$

Thus the proof is complete if we show that both $A_{n}$ and $R_{n}$ in (6.5) are bounded by $C K^{-\eta}$. First, since by Lemma $4.3, E\left|Z_{j, n}^{(1)}\right| \leq C\left|j-q_{0}\right|^{1 / 2}$, then

$$
A_{n} \leq C \sum_{j=0: j \neq q_{0}, q_{0}-1}^{\tilde{n}-1} \beta_{j}^{*}\left|j-q_{0}\right|^{1 / 2}
$$

where $\beta_{j}^{*}=\sup _{\psi: \psi=\left(\theta, \lambda_{q}\right) \in \Psi_{K}}\left\{u_{n}^{-1}(\psi) \beta_{j, 1}(\psi)\right\}$. To estimate $\beta_{j}^{*}$, note that for $\psi \in$ $\Psi_{K}, u_{n}(\psi) \equiv n\left\|\theta-\theta_{0}\right\|^{2}+|\bar{q}| \geq \max \left(K, n\left\|\theta-\theta_{0}\right\|^{2},|\bar{q}|\right)$. Then

$$
u_{n}(\psi)^{-1}\left(|| \theta-\theta_{0}||+\left|\lambda_{\bar{q}}\right|_{+}^{1 / 2}\right) \leq C K^{-1 / 2} n^{-1 / 2}
$$

and

$$
\begin{aligned}
u_{n}(\psi)^{-1}|\bar{q}|^{1-\varepsilon} & \leq K^{-\varepsilon} \quad \text { if } \psi \in A, \\
u_{n}(\psi)^{-1} & \leq n^{-1} \log ^{2} n \quad \text { if } \psi \in B .
\end{aligned}
$$

Using (6.7)-(6.8) in the definition (6.3) of $\beta_{j, 1}(\psi)$ we obtain that

$$
\beta_{j}^{*} \leq C\left(K^{-1 / 2} n^{-1 / 2}\left|j-q_{0}\right|^{-1}+K^{-\varepsilon}\left|j-q_{0}\right|^{-2+\varepsilon}\right)
$$

so that by $(6.6)$

$$
A_{n} \leq\left(K^{-1 / 2} n^{-1 / 2} \sum_{j=0: j \neq q_{0}}^{\tilde{n}-1}\left|j-q_{0}\right|^{-1 / 2}+K^{-\varepsilon} \sum_{j=0: j \neq q_{0}}^{\tilde{n}-1}\left|j-q_{0}\right|^{-3 / 2+\varepsilon}\right) \leq C K^{-\varepsilon} .
$$

It remains to examine $R_{n}$. Denoting

$$
\tilde{q}=\arg \max _{q=0, \ldots, \tilde{n}} \sum_{j=0: j \neq q_{0}, q_{0}-1}^{\tilde{n}-1} \beta_{j, q}^{*}\left|Z_{j, n}^{(1)}\right|, \quad \beta_{j, q}^{*}=\sup _{\theta: \psi=\left(\theta, \lambda_{q}\right) \in \Psi_{K}} u_{n}^{-1}(\psi) \beta_{j, 2}(\psi)
$$

we observe that

$$
R_{n} \leq E\left[\sum_{q=0}^{\tilde{n}-1} 1_{\{\tilde{q}=q\}} \sum_{j=0: j \neq q_{0}, q_{0}-1}^{\tilde{n}-1} \beta_{j, q}^{*}\left|Z_{j, n}^{(1)}\right|\right]
$$


Since (4.6)-(4.7) and (5.24) imply $\left|Z_{j, n}^{(1)}\right| \leq\left|Z_{j, n}^{(2)}\right|+\left|Z_{j, n}^{(3)}\right| \leq C\left|j-q_{0}\right|^{1 / 2} Z_{n}+$ $\tilde{Z}_{j, n}^{(2)}+\left|Z_{j, n}^{(3)}\right|$ we obtain

$$
R_{n} \leq C\left(E S_{n, 1}^{\prime}+E \sum_{q=0}^{\tilde{n}-1}\left[1_{\{\tilde{q}=q\}} S_{n, 2}(q)\right]+E \sum_{q=0}^{\tilde{n}-1}\left[1_{\{\tilde{q}=q\}} S_{n, 3}(q)\right]\right),
$$

where

$$
\begin{aligned}
S_{n, 1}^{\prime} & =Z_{n} \sup _{q=0, \ldots, \tilde{n}} B(q), \quad B(q)=\sum_{j=0: j \neq q_{0}, q_{0}-1} \beta_{j, q}^{*}\left|j-q_{0}\right|^{1 / 2}, \\
S_{n, 2}(q) & =\sum_{j=0: j \neq q_{0}, q_{0}-1}^{\tilde{n}-1} \beta_{j, q}^{*}\left|\tilde{Z}_{j, n}^{(2)}\right|, \quad S_{n, 3}(q)=\sum_{j=0: j \neq q_{0}, q_{0}-1}^{\tilde{n}-1} \beta_{j, q}^{*}\left|Z_{j, n}^{(3)}\right| .
\end{aligned}
$$

It remains to show that the terms on the right hand side of (6.9) are bounded by $C K^{-\eta}$. We first bound

$$
E S_{n, 1}^{\prime} \leq C \max _{q} B(q) E\left|Z_{n}\right| \leq C \max _{q} B(q) .
$$

We now estimate $B(q)$. Using (6.7)-(6.8) in (6.4), we obtain

$$
u_{n}^{-1}(\psi) \beta_{j, 2}(\psi) \leq C\left(\frac{K^{-\varepsilon}|\bar{q}|_{+}^{\varepsilon}}{\left|j-q_{0}\right|_{+}|j-q|_{+}}+\frac{n^{-\varepsilon}(\log n)^{2}}{\left|j-q_{0}\right|_{+}^{1-\varepsilon}|j-q|_{+}}\right)=: \nu_{j}(q) .
$$

Thus $\beta_{j, q}^{*} \leq \nu_{j}(q)$ and

$$
\begin{aligned}
B(q) \leq & C \sum_{j=0: j \neq q_{0}, q_{0}-1}^{\tilde{n}-1} \nu_{j}(q)\left|j-q_{0}\right|^{1 / 2} \\
\leq & C\left\{K^{-\varepsilon}|\bar{q}|^{\varepsilon} \sum_{j=0}^{\tilde{n}}\left|j-q_{0}\right|_{+}^{-1 / 2}|j-q|_{+}^{-1}\right. \\
& \left.\quad+n^{-\varepsilon}(\log n)^{2} \sum_{j=0}^{\tilde{n}}\left|j-q_{0}\right|_{+}^{-1 / 2+\varepsilon}|j-q|_{+}^{-1}\right\} \\
\leq & C\left\{K^{-\varepsilon}|\bar{q}|_{+}^{-1 / 2+2 \varepsilon}+n^{-\varepsilon}(\log n)^{2}|\bar{q}|_{+}^{-1 / 2+2 \varepsilon}\right\} \\
\leq & C K^{-\varepsilon}|\bar{q}|_{+}^{-1 / 2+2 \varepsilon}
\end{aligned}
$$

for sufficiently large $n$. Therefore

$$
E S_{n, 1}^{\prime} \leq C K^{-\varepsilon}
$$

To bound the 2nd and 3rd terms in (6.9), set $p_{q}=$ Prob $(\tilde{q}=q)$. By Hölder's inequality it follows that

$$
\begin{aligned}
\sum_{q=0}^{\tilde{n}}( & \left.E\left[1_{\{\tilde{q}=q\}} S_{n, 2}(q)\right]+E\left[1_{\{\tilde{q}=q\}} S_{n, 3}(q)\right]\right) \\
& \leq \sum_{q=0}^{\tilde{n}} p_{q}^{3 / 4}\left(\left(E S_{n, 2}(q)^{4}\right)^{1 / 4}+p_{q}^{1 / 2}\left(E S_{n, 3}(q)^{2}\right)^{1 / 2}\right)
\end{aligned}
$$




$$
\begin{aligned}
& \leq\left(\sum_{q=0}^{\tilde{n}} p_{q}\right)^{3 / 4}\left(\sum_{q=0}^{\tilde{n}} E S_{n, 2}(q)^{4}\right)^{1 / 4}+\left(\sum_{q=0}^{\tilde{n}} p_{q}\right)^{1 / 2}\left(\sum_{q=0}^{\tilde{n}} E S_{n, 3}(q)^{2}\right)^{1 / 2} \\
& =\left(\sum_{q=0}^{\tilde{n}} E S_{n, 2}(q)^{4}\right)^{1 / 4}+\left(\sum_{q=0}^{\tilde{n}} E S_{n, 3}(q)^{2}\right)^{1 / 2}
\end{aligned}
$$

since $\sum_{q=0}^{\tilde{n}} p_{q}=1$. To complete the proof, it suffices to show that for some $\eta>0$,

$$
\sum_{q=0}^{\tilde{n}} E S_{n, 2}(q)^{4} \leq C K^{-\eta}, \quad \sum_{q=0}^{\tilde{n}} E S_{n, 3}(q)^{2} \leq C K^{-\eta} .
$$

By Lemma 5.4, $E\left(\tilde{Z}_{j, n}^{(2)}\right)^{4} \leq C\left|j-q_{0}\right|^{2}$. Therefore

$$
E\left|\tilde{Z}_{j_{1}, n}^{(2)} \ldots \tilde{Z}_{j_{4}, n}^{(2)}\right| \leq\left(E\left(\tilde{Z}_{j_{1}, n}^{(2)}\right)^{4} \cdots E\left(\tilde{Z}_{j_{4}, n}^{(2)}\right)^{4}\right)^{1 / 4} \leq C\left(\prod_{i=1}^{4}\left|j_{i}-q_{0}\right|^{2}\right)^{1 / 4}
$$

and

$$
\begin{aligned}
E\left(S_{n, 2}(q)\right)^{4} & =\sum_{j_{1}, \ldots, j_{4}=0: j_{l} \neq q_{0}, q_{0}-1}^{\tilde{n}-1} \beta_{j_{1}, q}^{*} \cdots \beta_{j_{4}, q}^{*} E\left|\tilde{Z}_{j_{1}, n}^{(2)} \cdots \tilde{Z}_{j_{4}, n}^{(2)}\right| \\
& \leq C B(q)^{4} .
\end{aligned}
$$

Hence, (6.15) and (6.12) imply

$$
\sum_{q=0}^{\tilde{n}} E S_{n, 2}(q)^{4} \leq C K^{-4 \varepsilon} \sum_{q=0}^{\tilde{n}}\left|q-q_{0}\right|_{+}^{8 \varepsilon-2} \leq C K^{-4 \varepsilon} .
$$

By Lemma $4.3, E\left(Z_{j, n}^{(3)}\right)^{2} \leq C\left|j-q_{0}\right|^{2 p}$ with $p \in(1 / 3,1 / 2)$. Therefore

$$
E\left|Z_{j_{1}, n}^{(3)} Z_{j_{2}, n}^{(3)}\right| \leq\left(E\left(Z_{j_{1}, n}^{(3)}\right)^{2} E\left(Z_{j_{2}, n}^{(3)}\right)^{2}\right)^{1 / 2} \leq C\left(\left|j_{1}-q_{0}\right|\left|j_{2}-q_{0}\right|\right)^{p}
$$

and

$$
\begin{aligned}
E S_{n, 3}^{2}(q) & =\sum_{j_{1}, j_{2}=0: j_{1}, j_{2} \neq q_{0}, q_{0}-1}^{\tilde{n}-1} \beta_{j_{1}, q}^{*} \beta_{j_{2}, q}^{*} E\left|Z_{j_{1}, n}^{(3)} Z_{j_{2}, n}^{(3)}\right| \\
& \leq C\left(\sum_{j=0: j \neq q_{0}, q_{0}-1}^{\tilde{n}-1} \beta_{j, q}^{*}\left|j-q_{0}\right|^{p}\right)^{2} .
\end{aligned}
$$

Since $\beta_{j, q}^{*} \leq \nu_{j}(q)$, this and (6.11) imply

$$
E S_{n, 3}(q)^{2} \leq C\left\{K^{-\varepsilon}|\bar{q}|_{+}^{\varepsilon} \sum_{j=0}^{\tilde{n}}\left|j-q_{0}\right|_{+}^{-1+p}|j-q|_{+}^{-1}\right.
$$




$$
\begin{gathered}
\left.+n^{-\varepsilon}(\log n)^{2} \sum_{j=0}^{\tilde{n}}\left|j-q_{0}\right|_{+}^{-1+p+\varepsilon}|j-q|_{+}^{-1}\right\}^{2} \\
\leq C\left(K^{-\varepsilon}|\bar{q}|_{+}^{2 \varepsilon+p-1}+n^{-\varepsilon}(\log n)^{2}|\bar{q}|_{+}^{2 \varepsilon+p-1}\right)^{2} \leq C K^{-2 \varepsilon}|\bar{q}|_{+}^{4 \varepsilon+2 p-2} .
\end{gathered}
$$

Hence

$$
\sum_{q=0}^{\tilde{n}} E S_{n, 3}(q)^{2} \leq C K^{-2 \varepsilon} \sum_{q=0}^{\tilde{n}}\left|q-q_{0}\right|_{+}^{4 \varepsilon+2 p-2} \leq C K^{-2 \varepsilon}
$$

since $4 \varepsilon+2 p-2<-1$ for $p \in(1 / 3,1 / 2)$ and sufficiently small $\varepsilon>0$. Thus (6.14) holds.

LEMMA 6.2. Let $V_{n}(\psi)=1-2 \pi \sigma_{0}^{-2} k\left(\lambda_{q_{0}} ; \theta, \lambda_{q}\right)^{-1} I_{q_{0}}$. Under the assumptions of Theorem 3.1, there exists $\eta>0$ such that

$$
E \sup _{\psi \in \Psi_{K}}\left|u_{n}^{-1}(\psi) V_{n}(\psi)\right| \leq C K^{-\eta}
$$

where $0<C<\infty$ does not depend on $K, n$.

Proof. By A.1' or A.1, $k_{q_{0}}(\psi)^{-1} \leq C\left|\lambda_{\bar{q}}\right|^{\alpha}$. If $\left|\alpha-\alpha_{0}\right| \leq(\log n)^{-1}$ and $q \neq$ $q_{0}$ then $\left|\lambda_{\bar{q}}\right|^{\alpha-\alpha_{0}} \leq C$, and $k_{q_{0}}(\psi)^{-1} \leq C\left|\lambda_{\bar{q}}\right|^{\alpha_{0}} \leq C u_{n}(\psi)^{\alpha_{0}} n^{-\alpha_{0}}$. If $\left|\alpha-\alpha_{0}\right| \geq$ $(\log n)^{-1}$, then $u_{n}(\psi) \geq n\left\|\theta-\theta_{0}\right\|^{2} \geq n \log ^{-2} n$. Hence for $\psi \in \Psi_{K}$,

$u_{n}(\psi)^{-1}\left|V_{n}(\psi)\right| \leq C\left(u_{n}(\psi)^{\alpha_{0}-1} n^{-\alpha_{0}}+n^{-1} \log ^{2} n\right)\left(I_{q_{0}}+1\right) \leq C K^{-1+\alpha_{0}}\left(n^{-\alpha_{0}} I_{q_{0}}+1\right)$.

Since by standard arguments $E\left[n^{-\alpha_{0}} I_{q_{0}}\right] \leq C$, it follows that (6.16) holds with $\eta=1-\alpha_{0}$.

LEMMA 6.3. Let $b_{j}(\psi)$ be defined by (6.1). Under the assumptions of Lemma 6.1 , there exists $0<\varepsilon<1 / 2$, such that as $n \rightarrow \infty$, uniformly in $j=0, \ldots, \tilde{n}-1$ $\left(j \neq q_{0}, q_{0}-1\right)$

$$
\left|b_{j}(\psi)-b_{j+1}(\psi)\right| \leq C\left\{\begin{array}{r}
\frac{|| \theta-\theta_{0}||+\left|\lambda_{\bar{q}}\right|^{1 / 2}}{\left|j-q_{0}\right|}+|\bar{q}|_{+}^{1-\varepsilon}\left|j-q_{0}\right|^{-(2-\varepsilon)} \\
+|\bar{q}|_{+}\left|j-q_{0}\right|^{-1}|j-q|_{+}^{-1}, \\
\text { if }|| \theta-\theta_{0}|| \leq(\log n)^{-1} \\
n^{1-\varepsilon}\left|j-q_{0}\right|^{-(2-\varepsilon)}+n^{1-\varepsilon}\left|j-q_{0}\right|^{-(1-\varepsilon)}|j-q|_{+}^{-1} \\
\text { if }\left\|\theta-\theta_{0}\right\| \geq(\log n)^{-1}
\end{array}\right.
$$

where $0<C<\infty$ above does not depend on $n, \psi$, and moreover,

$$
1_{\left\{\omega_{0}<\pi\right\}}\left|b_{\tilde{n}}(\psi)\right|+1_{\left\{\omega_{0}>0\right\}}\left|b_{0}(\psi)\right| \leq C\left(|| \theta-\theta_{0} \|^{2}+\left|\lambda_{\bar{q}}\right|^{1 / 2}\right) .
$$


PRoOF. We prove first (6.17) in case $\left\|\theta-\theta_{0}\right\| \leq(\log n)^{-1}$. Write $\mid b_{j}(\psi)-$ $b_{j+1}(\psi)|=| v\left(\lambda_{j} ; \psi, \psi_{0}\right)-v\left(\lambda_{j+1} ; \psi, \psi_{0}\right) \mid$ where

$$
v\left(\lambda ; \psi, \psi_{0}\right)=k\left(\lambda ; \psi_{0}\right) k^{-1}(\lambda ; \psi) .
$$

Let $j \neq q, q \pm 1$. By the mean value theorem

$$
\left|v\left(\lambda_{j} ; \psi, \psi_{0}\right)-v\left(\lambda_{j+1} ; \psi, \psi_{0}\right)\right| \leq(2 \pi / n) \sup _{\xi \in\left(\lambda_{j}, \lambda_{j+1}\right)}\left|(d / d \xi) v\left(\xi ; \psi, \psi_{0}\right)\right| .
$$

Note that

$$
(\partial / \partial \xi) v\left(\xi ; \psi, \psi_{0}\right)=v\left(\xi ; \psi, \psi_{0}\right)\left|(\partial / \partial \xi) \log v\left(\xi ; \psi, \psi_{0}\right)\right| .
$$

By inequality (6.28) of Lemma 6.4 below,

$$
\begin{aligned}
& \sup _{\xi \in\left(\lambda_{j}, \lambda_{j+1}\right)} n^{-1}\left|(\partial / \partial \xi) \log v\left(\xi ; \psi, \psi_{0}\right)\right| \\
& \quad \leq C n^{-1}\left|(\partial / \partial \xi) \log v\left(\lambda_{j} ; \psi, \psi_{0}\right)\right| \\
& \quad \leq C\left(\left(|| \theta-\theta_{0}||+\left|\lambda_{\bar{q}}\right|^{1 / 2}\right)\left|j-q_{0}\right|^{-1}+|\bar{q}|\left|j-q_{0}\right|^{-1}|j-q|^{-1}\right) .
\end{aligned}
$$

Next we bound $v\left(\xi ; \psi, \psi_{0}\right)$. Relation (6.29) of Lemma 6.4 below implies that for $\xi \in\left(\lambda_{j}, \lambda_{j+1}\right)$ under Assumption A.1' or A.1 and $0<\omega_{0}<\pi$,

$$
\begin{aligned}
v\left(\xi ; \psi, \psi_{0}\right) & \leq C\left|\lambda_{j-q}\right|^{\alpha}\left|\lambda_{j-q_{0}}\right|^{-\alpha_{0}} \leq C\left|\frac{j-q}{j-q_{0}}\right|^{\alpha_{0}}\left|\lambda_{j-q}\right|^{\alpha-\alpha_{0}} \\
& \leq C\left|1-\frac{\bar{q}}{j-q_{0}}\right|^{\alpha_{0}}
\end{aligned}
$$

and under Assumption A.1 and $\omega_{0}=0, \pi$,

$$
v\left(\xi ; \psi, \psi_{0}\right) \leq C\left|\lambda_{j-q_{0}}^{2}-\lambda_{\bar{q}}^{2}\right|^{\alpha}\left|\lambda_{j-q_{0}}^{2}\right|^{-\alpha_{0}} \leq C\left|1-\left(\bar{q} /\left(j-q_{0}\right)\right)^{2}\right|^{\alpha_{0}}
$$

because $\left|\alpha-\alpha_{0}\right| \leq(\log n)^{-1}$ implies that $\left|\lambda_{j-q}\right|^{\alpha-\alpha_{0}} \leq C$ and $\left|\lambda_{j-q_{0}}^{2}-\lambda_{\bar{q}}^{2}\right|^{\alpha-\alpha_{0}} \leq C$.

If $|\bar{q}| /\left|j-q_{0}\right| \leq 2$, then (6.23)-(6.24) imply that $v\left(\lambda_{j} ; \psi, \psi_{0}\right) \leq C$, and from (6.20)-(6.22) it follows that

$$
\begin{aligned}
\left|b_{j}(\psi)-b_{j+1}(\psi)\right| \leq C\left(\left(|| \theta-\theta_{0}||\right.\right. & \left.+\left|\lambda_{\bar{q}}\right|^{1 / 2}\right)\left|j-q_{0}\right|^{-1} \\
& \left.+|\bar{q}|\left|j-q_{0}\right|^{-1}|j-q|^{-1}\right) .
\end{aligned}
$$

If $|\bar{q}| /\left|j-q_{0}\right|>2$, then (6.23)-(6.24) yield $v\left(\lambda_{j} ; \psi, \psi_{0}\right) \leq C\left(|\bar{q}| /\left|j-q_{0}\right|\right)^{1-\varepsilon}$ for small enough $\varepsilon>0$, and using (6.20)-(6.22) we obtain that

$$
\begin{aligned}
\left|b_{j}(\psi)-b_{j+1}(\psi)\right| \leq & C\left(|\bar{q}| /\left|j-q_{0}\right|\right)^{-(1-\varepsilon)} \\
& \times\left(\left(|| \theta-\theta_{0}||+\left|\lambda_{\bar{q}}\right|^{1 / 2}\right)\left|j-q_{0}\right|^{-1}+|\bar{q}|\left|j-q_{0}\right|^{-1}|j-q|^{-1}\right) \\
\leq & C|\bar{q}|^{1-\varepsilon}\left|j-q_{0}\right|^{-(2-\varepsilon)}
\end{aligned}
$$

since ||$\theta-\theta_{0}||+\left|\lambda_{\bar{q}}\right|^{1 / 2} \leq C$ by compactness of $\Theta$, and $|\bar{q}| /\left|j-q_{0}\right|>2$ implies $|\bar{q}| /|j-q| \leq 2$. From (6.25)-(6.26) we deduce (6.17). 
It remains to prove (6.17) in case $\left\|\theta-\theta_{0}\right\| \geq(\log n)^{-1}$. From (6.23) and (6.24) it follows that $v\left(\xi ; \psi, \psi_{0}\right) \leq C\left(n /\left|j-q_{0}\right|\right)^{1-\varepsilon}$ for arbitrarily small $\varepsilon>0$. Therefore (6.20)-(6.22) imply

$$
\begin{aligned}
& \left|b_{j}(\psi)-b_{j+1}(\psi)\right| \\
& \quad \leq C\left(n /\left|j-q_{0}\right|\right)^{1-\varepsilon}\left(\left(|| \theta-\theta_{0}||+\left|\lambda_{\bar{q}}\right|^{1 / 2}\right)\left|j-q_{0}\right|^{-1}\right. \\
& \left.\quad+|\bar{q}|\left|j-q_{0}\right|^{-1}|j-q|^{-1}\right) \\
& \quad \leq C\left(n^{1-\varepsilon}\left|j-q_{0}\right|^{-(2-\varepsilon)}+n^{1-\varepsilon}\left|j-q_{0}\right|^{-(1-\varepsilon)}|j-q|^{-1}\right)
\end{aligned}
$$

observing that ||$\theta-\theta_{0}||+\left|\lambda_{\bar{q}}\right|^{1 / 2}$ is bounded and $|\bar{q}|\left|j-q_{0}\right|^{-1}|j-q|^{-1} \leq 2(\mid j-$ $\left.\left.q_{0}\right|^{-1}+|j-q|^{-1}\right)$. From (6.27) we deduce (6.17).

We complete the proof by showing that (6.17) holds for $j=q, q \pm 1$ but $j \neq$ $q_{0}, q_{0}-1$. Note that $\left|b_{j}(\psi)-b_{j+1}(\psi)\right| \leq k_{j}\left(\psi_{0}\right) k_{j}(\psi)^{-1}+k_{j+1}\left(\psi_{0}\right) k_{j+1}(\psi)^{-1}$. If $\| \theta-\theta_{0}|| \leq(\log n)^{-1}$, then similarly as in (6.23)-(6.24) it follows that $\mid b_{j}(\psi)-$ $b_{j+1}(\psi) \mid \leq C$ and (6.17) holds.

If $\left\|\theta-\theta_{0}\right\| \geq(\log n)^{-1}$, then $k\left(\lambda_{q \pm 1} ; \psi_{0}\right) k\left(\lambda_{q \pm 1} ; \psi_{q}\right)^{-1} \leq C\left|\lambda_{\bar{q}}\right|^{-\alpha_{0}} \leq\left(n /|\bar{q}|_{+}\right)^{1-\varepsilon}$ for small enough $\varepsilon>0$. Therefore $\left|b_{n, j}(\psi)-b_{n, j+1}(\psi)\right| \leq C\left(n /|\bar{q}|_{+}\right)^{1-\varepsilon}$ which is bounded by the second term in (6.17) for $j=q, q \pm 1$ and $j \neq q_{0}, q_{0} \pm 1$.

Finally, (6.18) follows from relation (6.30) of Lemma 6.4 below.

LemMA 6.4. Let $v\left(\lambda ; \psi, \psi_{0}\right)$ be given by (6.19) and Assumptions A.1' or A.1 be satisfied. Then uniformly in $0<\lambda<\pi$ such that $\left|\lambda-\lambda_{q}\right| \geq \pi / n$ and $\left|\lambda-\lambda_{q_{0}}\right| \geq \pi / n$,

$$
\begin{aligned}
& \left|(\partial / \partial \lambda) \log v\left(\lambda ; \psi, \psi_{0}\right)\right| \\
& \quad \leq C\left(\frac{|| \theta-\theta_{0}||+\left|\lambda_{\bar{q}}\right|^{1 / 2}}{\left|\lambda-\lambda_{q_{0}}\right|}+\left|\lambda_{\bar{q}}\right|\left|\lambda-\lambda_{q_{0}}\right|^{-1}\left|\lambda-\lambda_{q}\right|^{-1}\right)
\end{aligned}
$$

where $C \in(0, \infty)$ does not depend on $n, \psi$, and

$$
\left|v\left(\lambda ; \psi, \psi_{0}\right)\right| \asymp\left\{\begin{array}{c}
\left|\lambda-\lambda_{q}\right|^{\alpha}\left|\lambda-\lambda_{q_{0}}\right|^{-\alpha_{0}}, \\
\text { if A.1 holds and } 0<\omega_{0}<\pi \text { or A.1' holds } \\
\left|\left(\lambda-\lambda_{q_{0}}\right)^{2}-\lambda_{\bar{q}}^{2}\right|^{\alpha}, \\
\text { if A.1 holds and } \omega_{0}=0, \pi
\end{array}\right.
$$

where $a_{n} \asymp b_{n}$ means that $c_{1} \leq a_{n} / b_{n} \leq c_{2}$ as $n \rightarrow \infty$ for some $0<c_{1}, c_{2}<\infty$. Moreover, for any $\delta>0$

$$
\left|v\left(\lambda ; \psi, \psi_{0}\right)-1\right| \leq C\left(|| \theta-\theta_{0}||+\left|\lambda_{\bar{q}}\right|^{1 / 2}\right) \quad \text { if }\left|\lambda-\lambda_{q_{0}}\right| \geq \delta .
$$

PRoOF. (6.29) follows from Assumptions A.1' or A.1, taking into account (3.9).

We now prove (6.28). Let A.1 $1^{\prime}$ hold. Then $\log k(\lambda ; \psi)=-\alpha \log \left|\lambda-\lambda_{q}\right|+$ $\log g(\lambda ; \psi)$. Since $(d / d x) \log |x|=1 / x \quad(x \neq 0)$, it follows that $(\partial / \partial \lambda) \log k(\lambda ; \psi)$ $=-\alpha\left(\lambda-\lambda_{q}\right)^{-1}+(\partial / \partial \lambda) \log g(\lambda ; \psi)$. Therefore

$$
\frac{\partial}{\partial \lambda} \log \left(\frac{k(\lambda ; \psi)}{k\left(\lambda ; \psi_{0}\right)}\right)=-\alpha\left(\lambda-\lambda_{q}\right)^{-1}+\alpha_{0}\left(\lambda-\lambda_{q_{0}}\right)^{-1}+h(\lambda ; \psi)-h\left(\lambda ; \psi_{0}\right)
$$


where $h(\lambda ; \psi)=(\partial / \partial \lambda) \log \left(g\left(\lambda ; \theta, \lambda_{q}\right)\right)$. (6.31) implies (6.28) noting that

$$
\begin{aligned}
\mid \alpha(\lambda & \left.-\lambda_{q}\right)^{-1}-\alpha_{0}\left(\lambda-\lambda_{q_{0}}\right)^{-1} \mid \\
& \leq\left|\left(\alpha_{0}-\alpha\right)\right| \lambda-\left.\lambda_{q_{0}}\right|^{-1}|+\alpha| \lambda_{\bar{q}}\left(\lambda-\lambda_{q}\right)^{-1}\left(\lambda-\lambda_{q_{0}}\right)^{-1} \mid \\
& \leq|| \theta-\theta_{0}||\left|\lambda-\lambda_{q_{0}}\right|^{-1}+\left|\lambda_{\bar{q}}\left(\lambda-\lambda_{q}\right)\right|^{-1}\left|\lambda-\lambda_{q_{0}}\right|^{-1}
\end{aligned}
$$

and, by Assumption A.1',

$$
\begin{aligned}
\left|h(\lambda ; \psi)-h\left(\lambda ; \psi_{0}\right)\right| & \leq C\left|h\left(\lambda ; \theta, \lambda_{q}\right)-h\left(\lambda ; \theta_{0}, \lambda_{q}\right)\right|+\left|h\left(\lambda ; \theta_{0}, \lambda_{q}\right)-h\left(\lambda ; \theta_{0}, \lambda_{q_{0}}\right)\right| \\
& \leq C\left(|| \theta-\theta_{0}||+\left|\lambda_{\bar{q}}\right|^{\beta}\right) \leq C\left(|| \theta-\theta_{0}||+\left|\lambda_{\bar{q}}\right|^{1 / 2}\right)
\end{aligned}
$$

where $\beta>1 / 2$ is the Lipschitz parameter.

Suppose that Assumption A.1 holds. By (3.9),

$$
\log k(\lambda ; \psi)= \begin{cases}-\alpha \log \left|\lambda-\lambda_{q}\right|-\alpha \log \left|\lambda+\lambda_{q}\right|+\log g_{1}(\lambda ; \psi), & \text { if } 0 \leq \lambda<\pi / 2, \\ -\alpha \log \left|\lambda-\lambda_{q}\right|-\alpha \log \left|\lambda+\lambda_{q}-2 \pi\right|+\log g_{2}(\lambda ; \psi), & \text { if } \pi / 2 \leq \lambda \leq \pi .\end{cases}
$$

(6.28) now follows by the same argument as in case of Assumption A.1' once it is observed that $\left|\lambda+\lambda_{q}-2 \pi\right| \geq\left|\lambda-\lambda_{q}\right|$ and $\left|\lambda+\lambda_{q}\right| \geq\left|\lambda-\lambda_{q}\right|$.

Finally, we show (6.30). Let $\left|\lambda-\lambda_{q_{0}}\right| \geq \delta$. Then under A. $1^{\prime}$ or A. $1 k^{-1}(\lambda ; \theta, \omega)$ and $k\left(\lambda ; \psi_{0}\right)$ are bounded. Therefore if $\left|\lambda_{\bar{q}}\right| \geq \delta / 2$, then

$$
\left|v\left(\lambda ; \psi, \psi_{0}\right)-1\right|=\left|1-k\left(\lambda ; \psi_{0}\right) k(\lambda ; \psi)^{-1}\right| \leq C \leq C(\delta / 2)^{-1 / 2}\left(|| \theta-\theta_{0}||+\left|\lambda_{\bar{q}}\right|^{1 / 2}\right) .
$$

If $\left|\lambda_{\bar{q}}\right| \leq \delta / 2$ then $\left|\lambda-\lambda_{q}\right| \geq\left|\lambda-\lambda_{q_{0}}\right|-\left|\lambda_{\bar{q}}\right| \geq \delta / 2$ and by A.1' or A.1,

$$
\begin{aligned}
\left|v\left(\lambda ; \phi, \phi_{0}\right)-1\right| & =k\left(\lambda ; \theta, \lambda_{q}\right)^{-1}\left|k\left(\lambda ; \theta, \lambda_{q}\right)-k\left(\lambda ; \theta_{0}, \lambda_{q_{0}}\right)\right| \\
& \leq C\left(\left|k\left(\lambda ; \theta, \lambda_{q}\right)-k\left(\lambda ; \theta_{0}, \lambda_{q}\right)\right|+\left|k\left(\lambda ; \theta_{0}, \lambda_{q}\right)-k\left(\lambda ; \theta_{0}, \lambda_{q_{0}}\right)\right|\right) \\
& \leq C\left(|| \theta-\theta_{0}||+\left|\lambda_{\bar{q}}\right|^{1 / 2}\right) .
\end{aligned}
$$

\section{Proof of (3.18).}

LEMMA 7.1. Let $T_{n}(\psi)$ be given by (3.15). Then under Assumptions A, as $n \rightarrow \infty$, there exists a constant $c>0$ such that for sufficiently large $K>0$,

$$
\inf _{\psi \in \Psi_{K}}\left\{u_{n}(\psi)^{-1} T_{n}(\psi)\right\} \geq c
$$

where $c$ does not depend on $K, n$ and $u_{n}(\psi)=n\left\|\theta-\theta_{0}\right\|^{2}+|\bar{q}|$.

Proof. Set $L\left(\lambda ; \psi, \psi_{0}\right)=-\log \left(v\left(\lambda ; \psi, \psi_{0}\right)\right)+v\left(\lambda ; \psi, \psi_{0}\right)-1$, where $v\left(\lambda ; \psi, \psi_{0}\right)$ is given by (6.19). $L$ is nonnegative for all $\lambda$ because $-\log x+x-1$ is, for all $x>0$. Rewrite

$$
\begin{aligned}
T_{n}(\psi)=\sum_{j\left(q_{0}, q\right)}{ }^{\prime}\left\{\frac{k\left(\lambda_{j} ; \psi_{0}\right)}{k\left(\lambda_{j} ; \psi\right)}-1\right\} & =\sum_{j\left(q_{0}, q\right)}{ }^{\prime} L\left(\lambda_{j} ; \psi, \psi_{0}\right)+\sum_{j\left(q_{0}, q\right)}{ }^{\prime} \log v\left(\lambda_{j} ; \psi, \psi_{0}\right) \\
& =: D_{n}(\psi)+F_{n}(\psi) .
\end{aligned}
$$


(7.1) follows if we show that as $n \rightarrow \infty$,

$$
D_{n}(\psi) \geq c u_{n}(\psi) \text { for some } c>0
$$

since by Lemma 7.3 below, $\left|F_{n}(\psi)\right| \leq(c / 2) u_{n}(\psi)$. We show that (7.2) holds in each of the following three, exhaustive, cases

$$
(\gamma 1)\left\|\theta-\theta_{0}\right\|^{2} \leq\left|\lambda_{\bar{q}}\right| \leq \delta ; \quad(\gamma 2)\left|\lambda_{\bar{q}}\right|+\left\|\theta-\theta_{0}\right\|^{2} \geq \delta ; \quad(\gamma 3)\left|\lambda_{\bar{q}}\right| \leq\left\|\theta-\theta_{0}\right\|^{2} \leq \delta
$$

for some $\delta>0$.

Case $(\gamma 1)$. Then $|\bar{q}| \geq u_{n}(\psi) / 4 \pi$ and (7.2) follows if $D_{n}(\psi) \geq c|\bar{q}|$. To show that note that by Lemma 7.2 below, for $\lambda \in A_{q}:=\left[\lambda_{q_{0}}+(3 / 4) \lambda_{\bar{q}}, \lambda_{q_{0}}+(7 / 8) \lambda_{\bar{q}}\right]$ and $\theta, q$ satisfying $(\gamma 1)$,

$$
\inf _{\lambda \in A_{q}}\left|v\left(\lambda ; \psi, \psi_{0}\right)-1\right| \geq c_{*}>0
$$

for small enough $\delta$. Then, since $L \geq 0$

$$
\begin{aligned}
D_{n}(\psi) & \geq \sum_{j=0: \lambda_{j} \in A_{q}}^{\tilde{n}} L\left(\lambda_{j}, \psi, \psi_{0}\right) \\
& \geq(|\bar{q}| / 8) \inf _{\lambda \in A_{q}} L\left(\lambda ; \psi, \psi_{0}\right) \geq(|\bar{q}| / 8) \inf _{|x-1| \geq c_{*}}(-\log x+x-1) \geq c|\bar{q}| .
\end{aligned}
$$

Case $(\gamma 2)$. Let $\varepsilon>0$. Under Assumptions A.1' or A.1, $\left|(\partial / \partial \lambda) L\left(\lambda ; \psi, \psi_{0}\right)\right|$ is bounded uniformly in $\lambda, \psi$ satisfying $\left|\lambda-\lambda_{q}\right| \geq \varepsilon,\left|\lambda-\lambda_{q_{0}}\right| \geq \varepsilon$. Therefore by standard arguments [see also the proof of (5.20)],

$$
D_{n}(\psi) \geq \sum_{j:\left|\lambda_{j}-\lambda_{q}\right| \geq \varepsilon,\left|\lambda_{j}-\lambda_{q_{0}}\right| \geq \varepsilon} L\left(\lambda_{j}, \psi, \psi_{0}\right)=(n / 2 \pi) d_{\varepsilon}(\psi)+\Delta_{n}(\psi)
$$

where

$$
d_{\varepsilon}(\psi)=\int_{0}^{\pi} 1_{\left\{\left|\lambda-\lambda_{q}\right| \geq \varepsilon,\left|\lambda-\lambda_{q_{0}}\right| \geq \varepsilon\right\}} L\left(\lambda ; \psi, \psi_{0}\right) d \lambda, \quad\left|\Delta_{n}(\psi)\right| \leq C<\infty
$$

and $C=C_{*}(\varepsilon)$ does not depend on $n$ and $\psi$. By (7.4), to prove (7.2) it suffices to show that

$$
d_{\varepsilon}(\psi) \geq c\left(\left|\lambda_{\bar{q}}\right|+\left\|\theta-\theta_{0}\right\|^{2}\right) .
$$

Since compactness of $\Theta$ implies $\left|\lambda_{\bar{q}}\right|+\left\|\theta-\theta_{0}\right\|^{2} \leq C<\infty$ for some $C>0$, (7.5) follows if

$$
d_{\varepsilon}(\psi) \geq c>0 \text { for some } c>0
$$

uniformly in $\psi$ satisfying $(\gamma 2)$. Set $d(\psi)=\int_{0}^{\pi} L\left(\lambda ; \psi, \theta_{0}, \omega_{0}\right) d \lambda$. Then

$$
d_{\varepsilon}(\psi) \geq \inf _{\psi} d(\psi)-\sup _{\psi}\left|d_{\varepsilon}(\psi)-d(\psi)\right|
$$

where $\inf _{\psi}$ and $\sup _{\psi}$ are taken over the compact set $\mathscr{I}_{\delta}=\{\psi=(\theta, \omega)$ : ||$\left.\theta-\left.\theta_{0}\right|^{2}+\left|\omega-\omega_{0}\right| \geq \delta / 4\right\} \cap \Theta \times[0, \pi]$. Under Assumptions A.1 $1^{\prime}$ or A.1, $d(\psi)$ is continuous. So it achieves its minimum, $d\left(\psi^{*}\right)>0$, on $\mathscr{I}_{\delta}$, since $L \geq 0$ by (3.3) for $\left\|\psi_{0}-\psi^{*}\right\| \geq \delta$. On the other hand, under Assumptions A.1' or A.1 we 
can choose $\varepsilon>0$ such that

$$
\sup _{\psi}\left|d_{\varepsilon}(\psi)-d(\psi)\right|<d\left(\psi^{*}\right) / 2 .
$$

Indeed, if A.1' holds then by (6.29) $L\left(\lambda ; \psi, \psi_{0}\right) \leq C\left(1+\log \left|\lambda-\lambda_{q}\right|+\log \left|\lambda-\lambda_{q_{0}}\right|\right.$ $\left.+\left|\lambda-\lambda_{q_{0}}\right|^{-\alpha_{0}}\right)$, and therefore

$$
\sup _{\psi}\left|d_{\varepsilon}(\psi)-d(\psi)\right| \leq \sup _{\psi \in \Psi_{K}} \int_{0}^{\pi} 1_{\left\{\left|\lambda-\lambda_{q}\right|<\varepsilon \text { or }\left|\lambda-\lambda_{q_{0}}\right|<\varepsilon\right\}} L\left(\lambda ; \psi, \psi_{0}\right) d \lambda
$$

can be made arbitrarily small by choosing sufficiently small $\varepsilon$, so that (7.7) holds. Under Assumption A.1, (7.7) follows using a similar argument. Hence (7.6) holds with $c=d\left(\psi^{*}\right) / 2$.

Case ( $\gamma 3$ ). As above it suffices to show (7.5). Under Assumptions A.1' or A.1, the Taylor expansion with respect to $\theta$ can be applied in (7.4), which leads to

$$
\begin{aligned}
d_{\varepsilon}(\psi) & =d_{\varepsilon}\left(\theta_{0}, \lambda_{q}\right)+\nabla d_{\varepsilon}\left(\theta_{0}, \lambda_{q}\right)\left(\theta-\theta_{0}\right)+\left(\theta-\theta_{0}\right)^{\prime} M\left(\theta-\theta_{0}\right) \\
& \geq \nabla d_{\varepsilon}\left(\theta_{0}, \lambda_{q}\right)\left(\theta-\theta_{0}\right)+\left(\theta-\theta_{0}\right)^{\prime} M\left(\theta-\theta_{0}\right)
\end{aligned}
$$

since $d_{\varepsilon}\left(\theta_{0}, \lambda_{q}\right) \geq 0$ by $L \geq 0$ and (7.4). The $i$ th element of the vector $\nabla d_{\varepsilon}\left(\theta_{0}, \lambda_{q}\right)$ is

$$
\left(\partial / \partial \theta_{i}\right) d_{\varepsilon}\left(\theta_{0}, \lambda_{q}\right)=\int_{0}^{\pi} 1_{\left\{\left|\lambda-\lambda_{q}\right| \geq \varepsilon,\left|\lambda-\lambda_{q_{0}}\right| \geq \varepsilon\right\}}\left(\partial / \partial \theta_{i}\right) L\left(\lambda ; \theta_{0}, \lambda_{q}, \psi_{0}\right) d \lambda,
$$

whereas the $(j, i)$ th element of the $p \times p$ matrix $M$ is

$$
\left(\partial^{2} / \partial \theta_{j} \partial \theta_{i}\right) d_{\varepsilon}\left(\theta_{*}^{(i)}, \lambda_{q}\right)=\int_{0}^{\pi} 1_{\left\{\left|\lambda-\lambda_{q}\right| \geq \varepsilon,\left|\lambda-\lambda_{q_{0}}\right| \geq \varepsilon\right\}}\left(\partial^{2} / \partial \theta_{j} \partial \theta_{i}\right) L\left(\lambda ; \theta_{*}^{(i)}, \lambda_{q}, \psi_{0}\right) d \lambda
$$

with $\left\|\theta_{*}^{(i)}-\theta_{0}\right\| \leq\left\|\theta-\theta_{0}\right\| \leq \delta^{1 / 2}$ under $(\gamma 3)$. Now if there exist $\varepsilon, \delta>0$ such that

$$
\begin{aligned}
\left(\theta-\theta_{0}\right)^{\prime} M\left(\theta-\theta_{0}\right) & \geq c|| \theta-\theta_{0} \|^{2}, \\
\left|\nabla d_{\varepsilon}\left(\theta_{0}, \lambda_{q}\right)\left(\theta-\theta_{0}\right)\right| & \leq c\left\|\theta-\theta_{0}\right\|^{2} / 2,
\end{aligned}
$$

then by (7.8) $d_{\varepsilon}(\psi) \geq\left(\theta-\theta_{0}\right)^{\prime} M\left(\theta-\theta_{0}\right)-\left|\nabla d_{\varepsilon}\left(\theta_{0}, \lambda_{q}\right)\left(\theta-\theta_{0}\right)\right| \geq c|| \theta-\theta_{0} \|^{2} / 2 \geq$ $c\left(\left\|\theta-\theta_{0}\right\|^{2}+\left|\lambda_{\bar{q}}\right|\right) / 4$ by $(\gamma 3)$, and so (7.5) follows. To verify (7.9), note that

$$
\left(\theta-\theta_{0}\right)^{\prime} M\left(\theta-\theta_{0}\right) \geq\left(\theta-\theta_{0}\right)^{\prime} \Omega\left(\theta-\theta_{0}\right)-\left|\left(\theta-\theta_{0}\right)^{\prime}(\Omega-M)\left(\theta-\theta_{0}\right)\right| .
$$

By (3.4), $\Omega>0$, so $\left(\theta-\theta_{0}\right) \Omega\left(\theta-\theta_{0}\right)^{\prime} \geq c\left\|\theta-\theta_{0}\right\|^{2}$ for some $c>0$. Under Assumptions A.1' or A.1 the $(j, i)$ th element of $M-\Omega$ is of form

$$
\begin{aligned}
\int_{0}^{\pi} & 1_{\left\{\left|\lambda-\lambda_{q}\right| \geq \varepsilon,\left|\lambda-\lambda_{q_{0}}\right| \geq \varepsilon\right\}} \frac{\partial^{2}}{\partial \theta_{j} \partial \theta_{i}} L\left(\lambda ; \theta_{*}^{(i)}, \lambda_{q}, \psi_{0}\right) d \lambda-\int_{0}^{\pi} \frac{\partial^{2}}{\partial \theta_{j} \partial \theta_{i}} L\left(\lambda ; \psi_{0}, \psi_{0}\right) d \lambda \\
= & \int_{0}^{\pi} 1_{\left\{\left|\lambda-\lambda_{q}\right| \geq \varepsilon,\left|\lambda-\lambda_{q_{0}}\right| \geq \varepsilon\right\}}\left(\frac{\partial^{2}}{\partial \theta_{j} \partial \theta_{i}} L\left(\lambda ; \theta_{*}^{(i)}, \lambda_{q}, \psi_{0}\right)-\frac{\partial^{2}}{\partial \theta_{j} \partial \theta_{i}} L\left(\lambda ; \psi_{0}, \psi_{0}\right)\right) d \lambda \\
& \quad-\int_{0}^{\pi} 1_{\left\{\left|\lambda-\lambda_{q}\right|<\varepsilon \text { or }\left|\lambda-\lambda_{q_{0}}\right|<\varepsilon\right\}} \frac{\partial^{2}}{\partial \theta_{j} \partial \theta_{i}} L\left(\lambda ; \psi_{0}, \psi_{0}\right) d \lambda,
\end{aligned}
$$


where each of the two integrals can be made arbitrarily small by choosing, respectively, $\varepsilon$ and $\delta$ sufficiently small. Thus, there exist $\varepsilon>0$ and $\delta>0$ such that $\|\Omega-M\| \leq c / 2$ and

$$
\left(\theta-\theta_{0}\right)^{\prime} M\left(\theta-\theta_{0}\right) \geq c\left\|\theta-\theta_{0}\right\|^{2}-\|\Omega-M\| \cdot\left\|\theta-\theta_{0}\right\|^{2} \geq(c / 2)\left\|\theta-\theta_{0}\right\|^{2},
$$

to prove (7.9).

It remains to prove (7.10). By Assumptions A.1' or A.1, the partial derivative $\nabla_{\theta} d_{\varepsilon}\left(\theta, \lambda_{q}\right)$ satisfies a Lipschitz condition in $\lambda$ of order $\beta>1 / 2$. Note that $\nabla_{\theta} L\left(\lambda ; \psi_{0}, \psi_{0}\right)=0$ and thus $\nabla_{\theta} d_{\varepsilon}\left(\theta_{0}, \lambda_{q_{0}}\right)=0$. Therefore

$$
\begin{aligned}
\left|\nabla_{\theta} d_{\varepsilon}\left(\theta_{0}, \lambda_{q}\right)\left(\theta-\theta_{0}\right)\right| & \leq\left\|\nabla d_{\varepsilon}\left(\theta_{0}, \lambda_{q}\right)-\nabla d_{\varepsilon}\left(\theta_{0}, \lambda_{q_{0}}\right)\right\| \cdot\left\|\theta-\theta_{0}\right\| \\
& \leq C(\varepsilon)\left|\lambda_{q}-\lambda_{q_{0}}\right|^{\beta}\left\|\theta-\theta_{0}\right\| \leq C(\varepsilon)\left\|\theta-\theta_{0}\right\|^{1+2 \beta} \\
& \leq C(\varepsilon) \delta^{(2 \beta-1) / 2}\left\|\theta-\theta_{0}\right\|^{2}
\end{aligned}
$$

since $\left|\lambda_{q}-\lambda_{q_{0}}\right|^{\beta} \leq\left\|\theta-\theta_{0}\right\|^{2 \beta} \leq \delta^{\beta}$ by $(\gamma 3)$. Choosing $\delta$ such that $C(\varepsilon) \delta^{(2 \beta-1) / 2}<$ $c / 2$ we obtain (7.10).

LEMMA 7.2. If $(\gamma 1)$ holds then (7.3) is valid.

PRoof. It suffices to show that uniformly in $\psi$ satisfying $(\gamma 1)$,

$$
\sup _{\lambda \in A_{q}} v\left(\lambda ; \psi, \psi_{0}\right) \leq c<1 .
$$

Let A.1' hold. Then $v\left(\lambda ; \psi, \psi_{0}\right)=\left|\lambda-\lambda_{q}\right|^{\alpha}\left|\lambda-\lambda_{q_{0}}\right|^{-\alpha_{0}} g\left(\lambda ; \psi_{0}\right) g(\lambda ; \psi)^{-1}$ and for $\lambda \in A_{q}$,

$$
v\left(\lambda ; \psi, \psi_{0}\right) \leq\left|\lambda_{\bar{q}} / 4\right|^{\alpha}\left|3 \lambda_{\bar{q}} / 4\right|^{-\alpha_{0}} g\left(\lambda ; \psi_{0}\right) / g(\lambda ; \psi) .
$$

Since $(\gamma 1)$ implies $\left|\alpha-\alpha_{0}\right|^{2} \leq|| \theta-\left.\theta_{0}\right|^{2} \leq\left|\lambda_{\bar{q}}\right| \leq \delta$, then $\left|\lambda_{\bar{q}} / 4\right|^{\alpha-\alpha_{0}}=\exp ((\alpha-$ $\left.\left.\alpha_{0}\right) \log \left(\left|\lambda_{\bar{q}} / 4\right|\right)\right)=1+O\left(\left|\alpha-\alpha_{0}\right| \log \left(\left|\alpha-\alpha_{0}\right|^{2}\right)\right)=1+O\left(\delta^{1 / 4}\right)$ and

$$
\left|\lambda_{\bar{q}} / 4\right|^{\alpha}\left|3 \lambda_{\bar{q}} / 4\right|^{-\alpha_{0}}=(1 / 3)^{\alpha_{0}}\left|\lambda_{\bar{q}} / 4\right|^{\alpha-\alpha_{0}}=(1 / 3)^{\alpha_{0}}\left(1+O\left(\delta^{1 / 4}\right)\right) .
$$

Under A.1',

$$
g\left(\lambda ; \psi_{0}\right) g(\lambda ; \psi)^{-1}=1+O\left(\left\|\theta-\theta_{0}\right\|+\left|\lambda_{\bar{q}}\right|^{\gamma}\right)=1+O\left(\delta^{1 / 2}\right) .
$$

Therefore from (7.12)-(7.14) it follows that $v\left(\lambda ; \psi, \psi_{0}\right) \leq(1 / 3)^{\alpha_{0}}\left(1+O\left(\delta^{1 / 4}\right)\right)<$ 1 assuming that $\delta$ is sufficiently small. Hence (7.11) holds.

Suppose that A. 1 holds. Let $0<\omega_{0}<\pi$. If $\delta$ is sufficiently small then the Gegenbauer spectral density (3.1) can be written in the form (3.7), and (7.11) can be shown using the same argument as above.

Let $\omega_{0}=0$. Then by (3.9),

$$
v\left(\lambda ; \psi, \psi_{0}\right)=\left|\lambda^{2}-\lambda_{q}^{2}\right|^{\alpha}|\lambda|^{-2 \alpha_{0}} g_{1}\left(\lambda ; \theta, \lambda_{q_{0}}\right) / g_{1}\left(\lambda ; \theta, \lambda_{q}\right) .
$$


Since for $q_{0}=0$ we have $\bar{q}=q$, then $\lambda \in A_{q}=\left[(3 / 4) \lambda_{q},(7 / 8) \lambda_{q}\right]$. Similarly to (7.14), it follows that $g_{1}\left(\lambda ; \theta, \lambda_{q}\right) / g_{1}\left(\lambda ; \theta_{0}, \lambda_{q_{0}}\right)=1+O\left(\delta^{1 / 2}\right)$. Therefore

$$
\begin{aligned}
v\left(\lambda ; \psi, \psi_{0}\right) & \leq\left|\lambda_{\bar{q}}^{2}-(3 / 4) \lambda_{\bar{q}}^{2}\right|^{\alpha}\left|(3 / 4) \lambda_{\bar{q}}\right|^{-2 \alpha_{0}}\left(1+O\left(\delta^{1 / 2}\right)\right) \\
& =(7 / 9)^{\alpha_{0}}\left|(7 / 16) \lambda_{\bar{q}}^{2}\right|^{\alpha-\alpha_{0}}\left(1+O\left(\delta^{1 / 2}\right)\right) .
\end{aligned}
$$

The same argument as that used to prove (7.13) implies that $\left|(7 / 16) \lambda_{\vec{q}}^{2}\right|^{\alpha-\alpha_{0}}=$ $1+O\left(\delta^{1 / 4}\right)$. Hence $v\left(\lambda ; \psi, \psi_{0}\right) \leq(7 / 9)^{\alpha_{0}}\left(1+O\left(\delta^{1 / 4}\right)\right)<1$ and (7.11) holds.

Let $\omega_{0}=\pi$. Then $\lambda_{q_{0}}=\pi$ and by (3.9), $v\left(\lambda ; \psi, \psi_{0}\right)=\left|(\lambda-\pi)^{2}-\left(\lambda_{q}-\pi\right)^{2}\right|^{\alpha} \mid \lambda-$ $\left.\pi\right|^{-2 \alpha_{0}} g_{2}\left(\lambda ; \theta, \lambda_{q}\right) / g_{2}\left(\lambda ; \theta_{0}, \lambda_{q_{0}}\right)$. Using a similar argument as in case $\omega_{0}=0$ we derive that for $\lambda \in A_{q}, v\left(\lambda ; \psi, \psi_{0}\right)$ satisfies (7.15) which proves (7.11).

LEMMA 7.3. Under the assumptions of Lemma 7.1, as $n \rightarrow \infty$,

$$
\sup _{\psi \in \Psi_{K}} u_{n}(\psi)^{-1}\left|\sum_{j\left(q, q_{0}\right)}{ }^{\prime} \log \left(k_{j}\left(\psi_{0}\right) / k_{j}(\psi)\right)\right| \leq C K^{-1 / 2} .
$$

Proof. Let $v\left(\lambda ; \psi, \psi_{0}\right)$ be given by (6.19). Then by (2.2), $\int_{0}^{n / 2} \log (v(2 \pi x / n$; $\left.\left.\psi, \psi_{0}\right)\right) d x=0$. Therefore

$$
\left|\sum_{j\left(q, q_{0}\right)}{ }^{\prime} \log \left(k_{j}\left(\psi_{0}\right) / k_{j}(\psi)\right)\right| \leq d_{n, 1}(\psi)+d_{n, 2}(\psi)+d_{n, 3}(\psi),
$$

where, setting $J_{q}=\left\{x \geq 0: x \notin[q-1, q+1] \cup\left[q_{0}-1, q_{0}+1\right]\right\}, J_{q}^{c}=[0, n / 2] \backslash J_{q}$,

$$
\begin{aligned}
& d_{n, 1}(\psi)=\int_{0}^{n / 2}\left|\log v\left(2 \pi[x] / n ; \psi, \psi_{0}\right)-\log v\left(2 \pi x / n ; \psi, \psi_{0}\right)\right| 1_{J_{q}} d x, \\
& d_{n, 2}(\psi)=\sum_{p \in\left\{\tilde{n}, q-1, q_{0}-1\right\} \backslash\left\{q, q_{0}\right\}}\left|\log v\left(\lambda_{p} ; \psi, \psi_{0}\right)\right|, \\
& d_{n, 3}(\psi)=\int_{0}^{n / 2}\left|\log v\left(2 \pi x / n ; \psi, \psi_{0}\right)\right| 1_{J_{q}^{c}} d x .
\end{aligned}
$$

It suffices to show that

$$
\sup _{\psi \in \Psi_{K}} u_{n}(\psi)^{-1} d_{n, i} \leq C K^{-1 / 2} \quad(i=1,2,3) .
$$

Using (6.28) to bound $\left|(\partial / \partial \xi) \log v\left(\xi ; \psi, \psi_{0}\right)\right|$, by the mean value theorem we obtain

$$
\begin{aligned}
d_{n, 1}(\psi) & \leq C \int_{0}^{n / 2} n^{-1} \sup _{\xi \in[2 \pi[x] / n, 2 \pi x / n]}\left|\frac{\partial}{\partial \xi} \log v\left(\xi ; \psi, \psi_{0}\right)\right| 1_{J_{q}} d x \\
& \leq C \int_{0}^{n / 2}\left(\left(|| \theta-\theta_{0}||+\left|\lambda_{\bar{q}}\right|^{1 / 2}\right)\left|x-q_{0}\right|^{-1}+|\bar{q}|\left|x-q_{0}\right|^{-1}|x-q|^{-1}\right) 1_{J_{q}} d x \\
& \leq C\left(|| \theta-\theta_{0}||+\left|\lambda_{\bar{q}}\right|^{1 / 2}\right) \log n+C \log |\bar{q}| .
\end{aligned}
$$

Thus, for $\psi \in \Psi_{K}$, as $n \rightarrow \infty$,

$$
u_{n}(\psi)^{-1}\left|d_{n, 1}(\psi)\right| \leq C n^{-1 / 2} \log n+C K^{-1 / 2} \leq 2 C K^{-1 / 2} .
$$


We now estimate $d_{n, 2}(\psi)$. (i) Suppose first that $\left|\alpha-\alpha_{0}\right| \geq(\log n)^{-1}$. Then it is easy to check that under Assumptions A.1 or A. $1^{\prime},\left|\log v\left(2 \pi p / n, \psi, \psi_{0}\right)\right| \leq$ $C \log n$. Thus, $d_{n, 2}(\psi) \leq C \log n \leq C n^{-1} \log n u_{n}(\psi)=o(1) u_{n}(\psi)$ and (7.18) holds.

(ii) If $\left|\alpha-\alpha_{0}\right|<(\log n)^{-1}$, then $\left|\lambda_{j}\right|^{\alpha-\alpha_{0}} \leq C$ uniformly in $j=1, \ldots, \tilde{n}$ and A.1 or A.1' imply that in $d_{n, 2}(\psi),\left|\log v\left(2 \pi p / n, \psi, \psi_{0}\right)\right| \leq C \log |\bar{q}|$. Thus $d_{n, 2}(\psi) \leq C \log |\bar{q}| \leq C \log u_{n}(\psi)$ and (7.18) holds.

To estimate $d_{n, 3}(\psi)$ note that when A.1 and $0<\omega_{0}<\pi$ hold or A.1' holds, (6.29) and the definition of $v\left(\lambda, \psi, \psi_{0}\right)$ imply $\mid \log \left(v\left(2 \pi x / n, \psi, \psi_{0}\right) \mid \leq C\left(\mid \alpha_{0}-\right.\right.$ $\left.\alpha|\log n+\log | x-q|+\log | x-q_{0} \mid+1\right)$. Then $d_{n, 3}(\psi) \leq C\left|\alpha_{0}-\alpha\right| \log n+C|\bar{q}|^{1 / 2}$ and

$$
\sup _{\psi \in \Psi_{K}} u_{n}(\psi)^{-1}\left|d_{n, 3}(\psi)\right| \leq C\left(n^{-1 / 2} \log n+K^{-1 / 2}\right) .
$$

When A.1 holds and $\omega_{0}=0, \pi$, using (6.29) it can be shown that (7.20) remains valid. Then (7.17)-(7.20) prove (7.16).

Acknowledgments. We are extremely grateful to Dr. Gilles Teyssiére, for carrying out the numerical experiment reported. We thank the referees and associate editor for helpful and constructive comments, which have improved the paper.

\section{REFERENCES}

ANDEL, J. (1986). Long-memory time series models. Kybernetika 22 105-123.

BLOOMFIELD, P. (1973). An exponential model for the spectrum of a scalar time series. Biometrika $60217-226$.

Box, G. E. P. and Jenkins, G. M. (1971). Time Series Analysis. Forecasting and Control. HoldenDay, San Francisco.

Chung, C. F. (1996a). Estimating a generalized long memory process. J. Econometrics 73 237-259.

Chung, C. F. (1996b). A generalized fractionally integrated autoregressive moving average process. J. Time Ser. Anal. 17 111-140.

DAHLHAus, R. (1989). Efficient parameter estimation for self-similar processes. Ann. Statist. 17 $1749-1766$.

FOX, R. and TAQQU, M. S. (1986). Large sample properties of parameter estimates for strongly dependent stationary Gaussian time series. Ann. Statist. 14 517-532.

GiRAITIS, L. and LEIPUS, R. (1995). A generalized fractionally differencing approach in longmemory modelling. Lithuanian Math. J. 35 65-81.

GIRAITIS, L. and SURGAILIS, D. (1990). A central limit theorem for quadratic forms in strongly dependent random variables and its application to asymptotical normality of Whittle's estimate. Probab. Theory Related Fields 86 87-104.

GrAY, H. L., ZHANG, N. and WoODWARD, W. A. (1989). On generalized fractional processes. J. Time Ser. Anal. $10233-257$.

Hannan, E. J. (1970). Multiple Time Series. Wiley, New York.

Hannan, E. J. (1973a). The estimation of frequency. J. Appl. Probab. 10 513-519.

Hannan, E. J. (1973b). The asymptotic theory of linear time series models. J. Appl. Probab. 10 130-145.

Hosking, J. R. M. (1981). Fractional differencing. Biometrika 68 165-176.

Hosoya, Y (1997). Limit theory with long-range dependence and statistical inference of related models. Ann. Statist. 25 105-137. 
Hidalgo, J. (1999). Semiparametric estimation of the location of the pole. Unpublished manuscript.

Robinson, P. M. (1978). Alternative models for stationary stochastic processes. Stochastic Process. Appl. 8 141-152.

Robinson, P. M. (1994). Efficient tests of nonstationary hypotheses. J. Amer. Statist. Assoc. 89 $1420-1437$.

Robinson, P. M. (1995a). Log-periodogram regression of time series with long range dependence. Ann. Statist. 23 1048-1072.

Robinson, P. M. (1995b). Gaussian semiparametric estimation of long range dependence. Ann. Statist. 23 1630-1661.

Stout, W. F. (1974). Almost Sure Convergence. Academic Press, New York.

YAJIMA, Y. (1996). Estimation of the frequency of unbounded spectral densities. In Proceedings of the Business and Economic Statistical Section 4-7. Amer. Statist. Assoc., Alexandria, VA.

DEPARTMENT OF ECONOMICS

LONDON SCHOOL OF ECONOMICS

HOUGHTON STREET

LONDON WC2A 2AE

UNITED KINGDOM

E-MAIL: L.Giraitis@lse.ac.uk

F.J.Hidalgo@lse.ac.uk

P.M.Robinson@lse.ac.uk 